ACCEPTED By The Astrophysical JOURnAL

Preprint typeset using LTEX style emulateapj v. 11/10/09

\title{
THE FIRST FERMI MULTIFREQUENCY CAMPAIGN ON BL LACERTAE: CHARACTERIZING THE LOW-ACTIVITY STATE OF THE EPONYMOUS BLAZAR
}

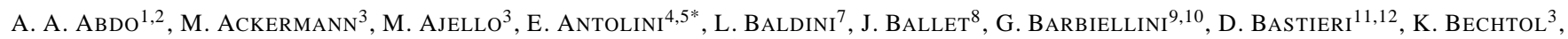

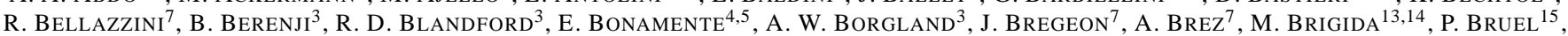
R. Buehler ${ }^{3}$, S. Buson ${ }^{11,12}$, G. A. CAliandro ${ }^{16}$, R. A. CAMERoN ${ }^{3}$, A. CANNON $^{17,18}$, P. A. CARAVEO ${ }^{19}$, S. CARRIGAN $^{12}$, J. M. CASANDJiAN $^{8}$,

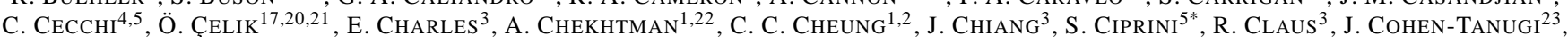
J. Conrad ${ }^{24,25,26}$, L. Costamante ${ }^{3}$, S. Cutini ${ }^{27}$, C. D. Dermer ${ }^{1}$, F. De Palma ${ }^{13,14}$, D. Donato $20,28 *$, E. Do Couto E Silva ${ }^{3}$, P. S. DRell ${ }^{3}$,

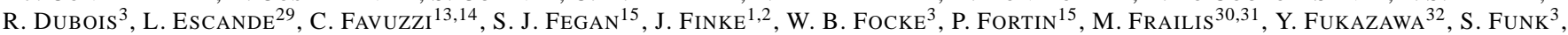
P. FusCo ${ }^{13,14}$, F. Gargano ${ }^{14}$, D. Gasparrini ${ }^{27}$, N. Gehrels ${ }^{17}$, S. Germani ${ }^{4,5}$, N. Giglietto ${ }^{13,14}$, F. Giordano ${ }^{13,14}$, M. Giroletti ${ }^{33}$,

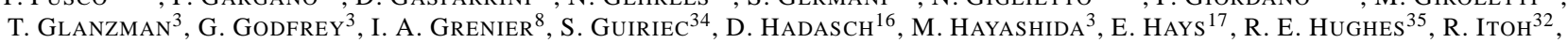
G. JóHANNESSON ${ }^{36}$, A. S. JOHNSON ${ }^{3}$, W. N. JOHNSON ${ }^{1}$, T. KAMAE ${ }^{3}$, H. KATAGIRI ${ }^{32}$, J. KATAOKA ${ }^{37}$, J. KNÖDLSEDER ${ }^{38}$, M. Kuss ${ }^{7}$, J. LANDE ${ }^{3}$,

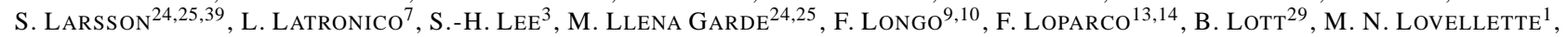

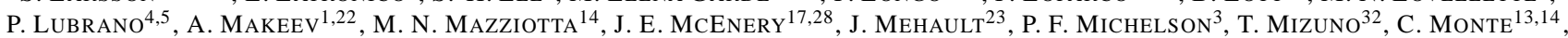
M. E. MonZani ${ }^{3}$, A. Morselli ${ }^{40}$, I. V. MoskalenKo ${ }^{3}$, S. Murgia ${ }^{3}$, T. NAKAMORI ${ }^{37}$, M. NAumanN-Godo ${ }^{8}$, S. NiShinO $^{32}$, P. L. NOlan $^{3}$,

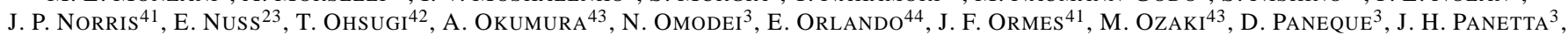

D. Parent ${ }^{1,22}$, V. Pelass ${ }^{23}$, M. PePe ${ }^{4,5}$, M. Pesce-Rollins ${ }^{7}$, F. Piron ${ }^{23}$, T. A. Porter ${ }^{3}$, S. Rainò ${ }^{13,14}$, R. Rando ${ }^{11,12}$, M. RazZANO ${ }^{7}$,

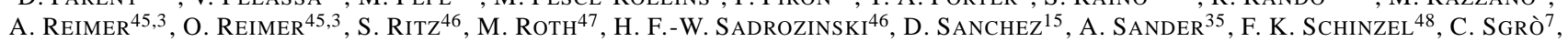

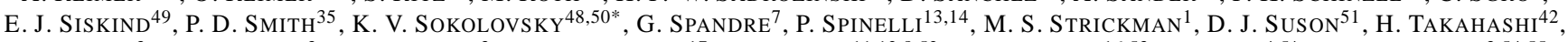

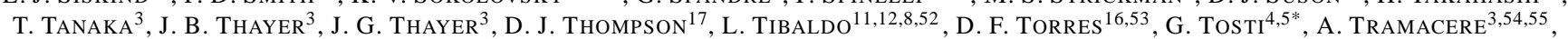

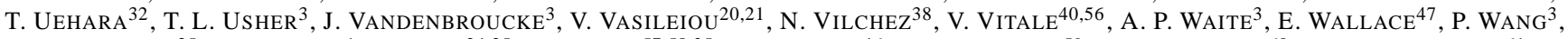

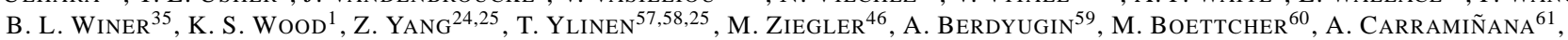

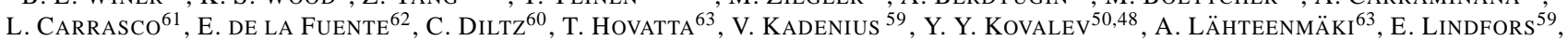

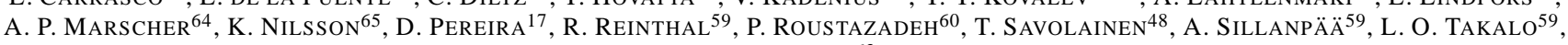
M. TORNIKOSKI ${ }^{63}$

Accepted by The Astrophysical Journal

\section{ABSTRACT}

We report on observations of BL Lacertae during the first 18 months of Fermi LAT science operations and present results from a 48-day multifrequency coordinated campaign from 2008 August 19 to 2008 October 7. The radio to gamma-ray behavior of BL Lac is unveiled during a low activity state thanks to the coordinated observations of radio-band (Metsähovi and VLBA), near-IR/optical (Tuorla, Steward, OAGH and MDM) and X-ray (RXTE and Swift ) observatories. No variability was resolved in gamma-rays during the campaign, and the brightness level was 15 times lower than the level of the 1997 EGRET outburst. Moderate and uncorrelated variability has been detected in UV and X-rays. The X-ray spectrum is found to be concave indicating the transition region between the low and high energy component of the spectral energy distribution (SED). VLBA observation detected a synchrotron spectrum self-absorption turnover in the innermost part of the radio jet appearing to be elongated and inhomogeneous, and constrained the average magnetic field there to be less than $3 \mathrm{G}$. Over the following months BL Lac appeared variable in gamma-rays, showing flares (in 2009 April and 2010 January). There is no evidence for correlation of the gamma-rays with the optical flux monitored from the ground in 18 months. The SED may be described by a single zone or two zone synchrotron self-Compton (SSC) model, but a hybrid SSC plus external radiation Compton (ERC) model seems preferred based on the observed variability and the fact that it provides a fit closest to equipartition.

Subject headings: Gamma rays: galaxies - BL Lacertae objects: individual: BL Lac - BL Lacertae objects: general - X-rays: galaxies - galaxies: jets - radiation mechanisms: non-thermal

* Corresponding authors: S. Ciprini, stefano.ciprini@pg.infn.it; E. Antolini, elisa.antolini@tiscali.it; D. Donato, davide.donato-1@nasa.gov; K. V. Sokolovsky, ksokolov@mpifr.de; G. Tosti, gino.tosti@pg.infn.it.

${ }^{1}$ Space Science Division, Naval Research Laboratory, Washington, DC 20375, USA

2 National Research Council Research Associate, National Academy of Sciences, Washington, DC 20001, USA

${ }^{3}$ W. W. Hansen Experimental Physics Laboratory, Kavli Institute for Particle Astrophysics and Cosmology, Department of Physics and SLAC National Accelerator Laboratory, Stanford University, Stanford, CA 94305, USA

${ }^{4}$ Istituto Nazionale di Fisica Nucleare, Sezione di Perugia, I-06123 Perugia, Italy

${ }^{5}$ Dipartimento di Fisica, Università degli Studi di Perugia, I-06123 Perugia, Italy Italy

${ }^{7}$ Istituto Nazionale di Fisica Nucleare, Sezione di Pisa, I-56127 Pisa,

${ }^{8}$ Laboratoire AIM, CEA-IRFU/CNRS/Université Paris Diderot, Service d'Astrophysique, CEA Saclay, 91191 Gif sur Yvette, France

${ }^{9}$ Istituto Nazionale di Fisica Nucleare, Sezione di Trieste, I-34127 Trieste, Italy

${ }^{10}$ Dipartimento di Fisica, Università di Trieste, I-34127 Trieste, Italy

${ }^{11}$ Istituto Nazionale di Fisica Nucleare, Sezione di Padova, I-35131 Padova, Italy

12 Dipartimento di Fisica “G. Galilei”, Università di Padova, I-35131
Padova, Italy

13 Dipartimento di Fisica “M. Merlin” dell’Università e del Politecnico di Bari, I-70126 Bari, Italy

${ }^{14}$ Istituto Nazionale di Fisica Nucleare, Sezione di Bari, 70126 Bari, Italy

${ }^{15}$ Laboratoire Leprince-Ringuet, École polytechnique, CNRS/IN2P3, Palaiseau, France

${ }^{16}$ Institut de Ciencies de l'Espai (IEEC-CSIC), Campus UAB, 08193 Barcelona, Spain

${ }^{17}$ NASA Goddard Space Flight Center, Greenbelt, MD 20771, USA

18 University College Dublin, Belfield, Dublin 4, Ireland

${ }^{19}$ INAF-Istituto di Astrofisica Spaziale e Fisica Cosmica, I-20133 Milano, Italy

${ }^{20}$ Center for Research and Exploration in Space Science and Technology (CRESST) and NASA Goddard Space Flight Center, Greenbelt, MD 20771, USA

${ }^{21}$ Department of Physics and Center for Space Sciences and Technology, University of Maryland Baltimore County, Baltimore, MD 21250 , USA

${ }^{22}$ George Mason University, Fairfax, VA 22030, USA

${ }^{23}$ Laboratoire de Physique Théorique et Astroparticules, Université Montpellier 2, CNRS/IN2P3, Montpellier, France

${ }^{24}$ Department of Physics, Stockholm University, AlbaNova, SE-106 91 Stockholm, Sweden

${ }^{25}$ The Oskar Klein Centre for Cosmoparticle Physics, AlbaNova, SE- 


\section{INTRODUCTION}

BL Lacertae (BL Lac, S4 2200+42, OY 401, B3 2200+420, 1ES 2200+420, 3EG J2202+4217, 1FGL J2202.8+4216) at redshift $z=0.0686$ (e.g., Vermeulen et al. 1995) was historically the prototype of the class of active galactic nuclei (AGN) for which BL Lac has become the eponym. It is categorized as an intermediate synchrotron peaked (ISP) BL Lac object. This is based on the latest HSP/ISP/LSP classification

10691 Stockholm, Sweden

${ }^{26}$ Royal Swedish Academy of Sciences Research Fellow, funded by a grant from the K. A. Wallenberg Foundation

${ }^{27}$ Agenzia Spaziale Italiana (ASI) Science Data Center, I-00044 Frascati (Roma), Italy

28 Department of Physics and Department of Astronomy, University of Maryland, College Park, MD 20742, USA

${ }^{29}$ Université Bordeaux 1, CNRS/IN2p3, Centre d'Études Nucléaires de Bordeaux Gradignan, 33175 Gradignan, France

${ }^{30}$ Dipartimento di Fisica, Università di Udine and Istituto Nazionale di Fisica Nucleare, Sezione di Trieste, Gruppo Collegato di Udine, I-33100 Udine, Italy

31 Osservatorio Astronomico di Trieste, Istituto Nazionale di Astrofisica, I-34143 Trieste, Italy

32 Department of Physical Sciences, Hiroshima University, HigashiHiroshima, Hiroshima 739-8526, Japan

33 INAF Istituto di Radioastronomia, 40129 Bologna, Italy

${ }^{34}$ Center for Space Plasma and Aeronomic Research (CSPAR), University of Alabama in Huntsville, Huntsville, AL 35899, USA

35 Department of Physics, Center for Cosmology and Astro-Particle Physics, The Ohio State University, Columbus, OH 43210, USA

${ }^{36}$ Science Institute, University of Iceland, IS-107 Reykjavik, Iceland

${ }^{37}$ Research Institute for Science and Engineering, Waseda University, 3-4-1, Okubo, Shinjuku, Tokyo, 169-8555 Japan

38 Centre d'Étude Spatiale des Rayonnements, CNRS/UPS, BP 44346, F-30128 Toulouse Cedex 4, France

39 Department of Astronomy, Stockholm University, SE-106 91 Stockholm, Sweden

${ }^{40}$ Istituto Nazionale di Fisica Nucleare, Sezione di Roma "Tor Vergata", I-00133 Roma, Italy

${ }^{41}$ Department of Physics and Astronomy, University of Denver, Denver, CO 80208, USA

${ }^{42}$ Hiroshima Astrophysical Science Center, Hiroshima University, Higashi-Hiroshima, Hiroshima 739-8526, Japan

43 Institute of Space and Astronautical Science, JAXA, 3-1-1 Yoshinodai, Sagamihara, Kanagawa 229-8510, Japan

${ }^{44}$ Max-Planck Institut für extraterrestrische Physik, 85748 Garching, Germany

${ }^{45}$ Institut für Astro- und Teilchenphysik and Institut für Theoretische Physik, Leopold-Franzens-Universität Innsbruck, A-6020 Innsbruck, Austria

${ }^{46}$ Santa Cruz Institute for Particle Physics, Department of Physics and Department of Astronomy and Astrophysics, University of California at Santa Cruz, Santa Cruz, CA 95064, USA

47 Department of Physics, University of Washington, Seattle, WA 98195-1560, USA

${ }^{48}$ Max-Planck-Institut für Radioastronomie, Auf dem Hügel 69, 53121 Bonn, Germany

${ }^{49}$ NYCB Real-Time Computing Inc., Lattingtown, NY 11560-1025, USA

${ }^{50}$ Astro Space Center of the Lebedev Physical Institute, 117810 Moscow, Russia

${ }^{51}$ Department of Chemistry and Physics, Purdue University Calumet, Hammond, IN 46323-2094, USA

${ }^{52}$ Partially supported by the International Doctorate on Astroparticle Physics (IDAPP) program

${ }^{53}$ Institució Catalana de Recerca i Estudis Avançats (ICREA), Barcelona, Spain

${ }^{54}$ Consorzio Interuniversitario per la Fisica Spaziale (CIFS), I-10133 Torino, Italy

55 INTEGRAL Science Data Centre, CH-1290 Versoix, Switzerland

56 Dipartimento di Fisica, Università di Roma "Tor Vergata", I-00133 Roma, Italy

57 Department of Physics, Royal Institute of Technology (KTH), AlbaNova, SE-106 91 Stockholm, Sweden proposed by Abdo et al. (2010a) to replace the older HBLIBL-LBL scheme (Padovani \& Giommi 1995; Fossati et al. 1998). BL Lac occasionally showed peculiar behavior that has questioned both this classification and a simple interpretation of its broad-band emission in terms of synchrotron and synchrotron self-Compton (SSC) emission produced by a single blob. In fact, BL Lac is a source that has shown quite complex and distinct X-ray spectral behavior (Madejski et al. 1999; Ravasio et al. 2002; Böttcher \& Reimer 2004) and during several epochs broad $\mathrm{H} \alpha$ and $\mathrm{H} \beta$ emission lines with luminosity ( $\sim 10^{41} \mathrm{erg} \mathrm{s}^{-1}$ ) comparable to those of type I Seyfert galaxies (Vermeulen et al. 1995; Corbett et al. 1996, 2000). There is evidence for an increase by $\sim 50 \%$ in ten years of the broad $\mathrm{H} \alpha$ line and an underluminous broad line region (BLR), compared to other AGN. The narrow lines and radio luminosities of BL Lac match those of of low-excitation and miniature radio galaxies (Capetti et al. 2010). Long term radio-optical flux density monitoring and several multiwavelength campaigns have been carried out on BL Lac, like the past campaigns of the Whole Earth Blazar Telescope (WEBT, Villata et al. 2002a b) dedicated to this source, providing very complete datasets (for example, Bloom et al. 1997; Sambruna et al. 1999; Madejski et al. 1999; Ravasio et al. 2003; Böttcher et al. 2003; Villata et al. 2002a, 2004a b, 2009; Raiteri et al. 2009, 2010; Marscher et al. 2008). In particular in summer 1997 BL Lac showed the largest optical outburst ever recorded in almost 33 years (Nesci et al. 1998; Tosti et al. 1999; Villata et al. 2004b).

In the X-ray band the two broad components of the spectral energy distribution (SED) are overlapping and the radiation at this band is at times dominated by the high-energy end of the synchrotron emission, while at other occasions it is dominated by the low-frequency portion of the high-energy component (e.g., Madejski et al. 1999; Ravasio et al. 2002). The rapid X-ray variability is mainly restricted to the low-energy excess portion of the X-ray spectrum, presumably produced by synchrotron radiation (Ravasio et al. 2002, 2003). The X-ray variability of BL Lac, except for a few major flaring events, has a log-normal distribution (Giebels \& Degrange 2009), meaning that the emission is a multiplicative product of a large number of independent random events.

Gamma-ray observations by EGRET resulted in several detections (Figure 1). Observations after 1995 resulted in an average $\gamma$-ray flux above $100 \mathrm{MeV}$ of $(40 \pm 12) \times 10^{-8}$ ph $\mathrm{cm}^{-2} \mathrm{~s}^{-1}$ (Catanese et al. 1997) and in a flare in 1997 at a level $(171 \pm 42) \times 10^{-8} \mathrm{ph} \mathrm{cm}^{-2} \mathrm{~s}^{-1}$ with $10.2 \sigma$ significance (Bloom et al. 1997). Correlated $\gamma$-rays and optical flaring emission were observed during the EGRET era (Bloom et al. 1997). Very high energy (VHE) $\gamma$-ray emission was claimed by the Crimean GT-48 (Neshpor et al.2001)

${ }^{58}$ School of Pure and Applied Natural Sciences, University of Kalmar, SE-391 82 Kalmar, Sweden

${ }^{59}$ Tuorla Observatory, University of Turku, FI-21500 Piikkiö, Finland

${ }^{60}$ Department of Physics and Astronomy, Ohio University, Athens, $\mathrm{OH}$ 45701, USA

${ }^{61}$ Instituto Nacional de Astrofísica, Óptica y Electrónica, Tonantzintla, Puebla 72840, Mexico

62 Instituto de Astronomía y Meteorología, CUCEI, Universidad de Guadalajara, 44130 Guadalajara , Jalisco, Mexico

${ }^{63}$ Aalto University Metsähovi Radio Observatory, FIN-02540 Kylmala, Finland

${ }^{64}$ Institute for Astrophysical Research, Boston University, Boston, MA 02215, USA

${ }^{65}$ Finnish Centre for Astronomy with ESO (FINCA), University of Turku, FI-21500 Piikkiö, Finland 


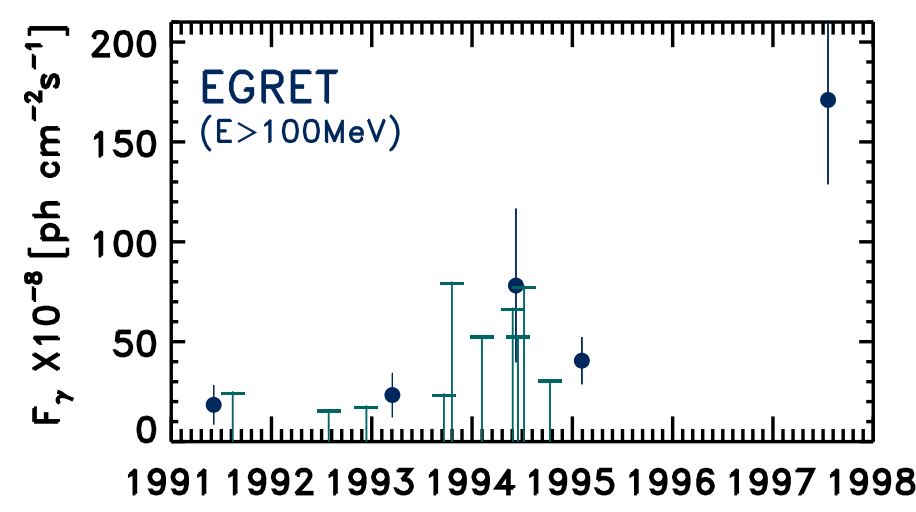

FIG. 1.- Gamma-ray light curve of BL Lac obtained by EGRET in the period 1991-1997. Data points have source detection significance above $4 \sigma$ (Nandikotkur et al. 2007).

and HEGRA (Kranich 2003) atmospheric Cherenkov telescopes. A significant $(>5 \sigma)$ detection above $200 \mathrm{GeV}$ was made only by the MAGIC telescope in 2005 (Albert et al. 2007). Gamma-ray emission was explained with the requirement of Comptonization of external-jet photons (externalradiation Compton, ERC) in addition to the SSC (in-jet) emission (Madejski et al. 1999; Böttcher \& Bloom 2000). Superluminal motion of $\beta_{\text {app }}$ up to $(10.57 \pm 0.74)$ has been observed in this object (Lister et al. 2009; Denn et al. 2000). Following results of past multifrequency campaigns, possibly distinct VLBI jet structures are assumed to contribute, sometimes with delays, to the radio flux density light curves, and sometimes are suggested to be responsible for optical to $\mathrm{TeV}$ $\gamma$-ray flares (for example, Bach et al. 2006; Marscher et al. 2008).

In this paper, we report on LAT observations of BL Lac during the first 18 months of Fermi science operations (from 2008 August 04 to 2010 February 04). The LAT's reasonably uniform exposure, high sensitivity, and continuous sky monitoring make it an excellent instrument around which to organize a simultaneous multifrequency campaign. The so-called Fermi planned intensive campaign (PIC) dedicated to BL Lac was performed in 2008 August 19 - 2008 October 7 (MJD 54697.8 - 54746), during roughly the first two months of science operations, irrespective of the brightness of the source. This was part of a series of Fermi-LAT-collaboration PICs (Tosti 2007; Thompson 2007), involving observing proposals submitted to the RXTE (Cycle 12) and Swift (Cycle 4) Xray satellites, and organized in advance of the Fermi launch. This ensured access to the facilities allowing the best multiwavelength (MW) coverage. The aim of the campaign is to shed light for the first time on the broad-band radio-to- $\gamma$-ray SED, including the high energy (X-ray and $\gamma$-ray) behavior, during a low activity phase of the source. The 18-month LAT light curve shows that BL Lac was variable in $\gamma$-rays for most of these 18 months, with the exception of the first 2 months, which corresponds to the period of the MW campaign. In section 2 the light curve by Fermi -LAT during the first 18 months of survey and simultaneous optical long-term monitoring data are presented joined to the analysis of the $\gamma$-ray spectrum. In section 3 the multiwaveband radio-band to X-ray flux density and parsec-scale radio structure observations collected during the campaign period are reported and discussed. The assembled spectral energy distribution and modeling are reported in section 3.4 and conclusions in section 4 .

A $\Lambda$ CDM cosmology with values given within $1 \sigma$ of the WMAP results (Komatsu et al. 2009), namely $\Omega_{m}=0.27$, $\Omega_{\Lambda}=0.73$, and $H_{0}=71 \mathrm{~km} \mathrm{~s}^{-1} \mathrm{Mpc}^{-1}$ is used.

\section{GAMMA-RAY OBSERVATIONS AND RESULTS BY FERMI-LAT}

\subsection{LAT analysis and observations}

The Large Area Telescope (LAT), on board the Fermi Gamma-ray Space Telescope (Atwood et al. 2009), is a pair-conversion $\gamma$-ray telescope, sensitive to photon energies from about $20 \mathrm{MeV}$ up to $>300 \mathrm{GeV}$. It consists of a tracker (composed of two sections, front and back, with different angular resolutions), a calorimeter and an anticoincidence system to reject the charged-particle background. The LAT has a large peak effective area $\left(\sim 8000 \mathrm{~cm}^{2}\right.$ for $1 \mathrm{GeV}$ photons in the event class considered here), viewing $\approx 2.4 \mathrm{sr}$ of the sky with angular resolution $(68 \%$ containment radius) better than $\approx 1^{\circ}$ at $E=1 \mathrm{GeV}$. The large field of view, improved effective area and sensitivity and the survey nominal mode make Fermi-LAT an optimal all-sky hunter for high-energy flares and an unprecedented monitor of $\gamma$-ray sources. The data set used in this paper was collected during the first 18 months of Fermi science obserations, from 2008 August 4 to 2010 February 4 (about 550 days, 78 weeks from MJD 54682.7 to 55232.9, as shown in the weekly light curve in Figure 2, upper panel). This interval includes the period chosen for the first Fermi MW campaign on BL Lac (2008, Aug. 19 - Oct. 7, MJD 54697.8-54746, i.e. about 48 days, indicated by the line in Figure 2, upper panel). Fermi -LAT data analysis was performed with the standard Fermi -LAT ScienceTools software package using version v9r15p5. Only events belonging to the "Diffuse" class in the energy range $0.1-100 \mathrm{GeV}$ were used in the analysis. Instrument response functions (IRFs) used were P6_V3_DIFFUSE. In order to provide protection against significant background contamination by Earth-limb $\gamma$ rays, all events with zenith angles $>105^{\circ}$ were excluded.

The 18-month light curve was built using 1-week time bins (Figures 2 and 4). For each time bin the integrated flux $(\mathrm{E}>100 \mathrm{MeV})$ values were computed using the maximum-likelihood algorithm implemented in the science tool gtlike. For each time bin we analyzed a region of interest (RoI) of $12^{\circ}$ in radius, centered on the position of the source. All point sources listed in the 1FGL (1-year) LAT catalog (Abdo et al. 2010b) within $19^{\circ}$ from BL Lacertae and having test statistid $\mathrm{TS}>50$ and fluxes above $10^{-8} \mathrm{ph}$ $\mathrm{cm}^{-2} \mathrm{~s}^{-1}$ were included in the RoI model using a powerlaw spectrum $\left(d N / d E \propto E^{-\Gamma}\right.$, where $\Gamma$ is the photon index $)$. The isotropic background (the sum of the residual instrumental background and extragalactic diffuse $\gamma$-ray background) was included in the RoI model using the standard model file isotropic_iem_v02.txt 68 , and the Galactic diffuse emission was included in the modeling using the file and the standard file gll_iem_v02.fit. In the final light curve computation the photon index value was frozen to the value resulting from the likelihood analysis on the entire period. For each time bin, if the TS value for the source was $T S<4$ or the number of model predicted photons $N_{\text {pred }}<10$, the value

\footnotetext{
66 http://fermi.gsfc.nasa.gov/ssc/data/analysis/documentation

67 The test statistic is defined as $T S=2 \Delta \log$ (Likelihood) between models with and without the source and it is a measure of the source significance (Mattox et al. 1996).

${ }^{68}$ http://fermi.gsfc.nasa.gov/ssc/data/access/lat/BackgroundM
} 


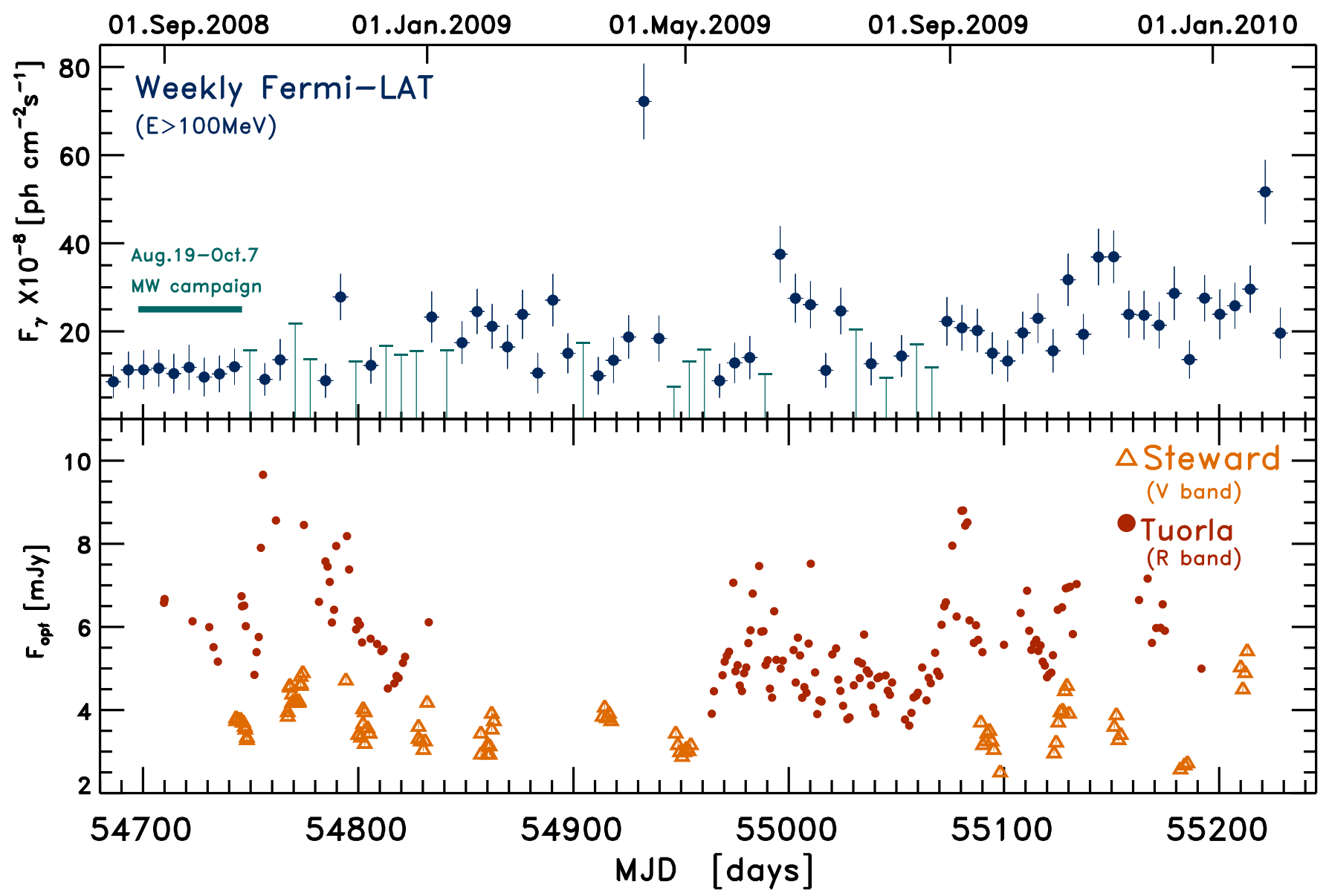

FIG. 2.- Top panel: 18-month weekly integrated flux $(E>100 \mathrm{MeV})$ light curve of BL Lac obtained obtained from 2008, Aug. 04 to 2010 , Feb. 04 . Bottom panel: superposed optical V-band and R-band observed flux (not corrected for interstellar absorption and host galaxy contribution) light curves obtained respectively at the Steward Observatory, Kitt Peak, Arizona, USA, and Tuorla Obs

of the fluxes were replaced by the 2- $\sigma$ upper limits. All errors reported in the figures or quoted in the text are 1- $\sigma$ statistical errors. The estimated relative systematic uncertainty on the flux, according to Abdo et al. (2010b) and reflecting the relative systematic uncertainty on effective area, is set to $10 \%$ at $100 \mathrm{MeV}, 5 \%$ at $500 \mathrm{MeV}$ and $20 \%$ at $10 \mathrm{GeV}$.

\subsection{Gamma-ray spectral and temporal behavior}

The $\gamma$-ray spectral analysis of BL Lacertae was performed both for the entire 18-month period, and for the 48 days of the campaign. These spectra are shown in Figure 3. The flux value for each energy bin and for the two periods are reported in Table 1. The most energetic photon observed within the $95 \%$ point spread function containment radius of the source for the 18-month period had energy $70 \mathrm{GeV}$, while for the campaign period the highest energy photon had $10 \mathrm{GeV}$. The energy-spectrum binning was built requiring $T S>50$ and/or model-predicted source photons $>8$, except for the last bin, which had an upper limit rather than a detection. The 18-month spectrum results in an integrated average flux of $(19.85 \pm 0.40) \times 10^{-8} \mathrm{ph} \mathrm{cm}^{-2} \mathrm{~s}^{-1}$, in the $0.1-100 \mathrm{GeV}$ range, with $T S=2375$ and photon index $\Gamma=2.38 \pm 0.01$, while the value for the 48-day interval of the multifrequency campaign, $(11.5 \pm 2.7) \times 10^{-8} \mathrm{ph} \mathrm{cm}^{-2} \mathrm{~s}^{-1}$, and the photon index slightly flatter $(\Gamma=2.27 \pm 0.10)$, with a $T S=111$.

In order to quantify the departure of the 18-month spectrum from a power law shape, we use a spectral curvature index $\mathcal{C}$

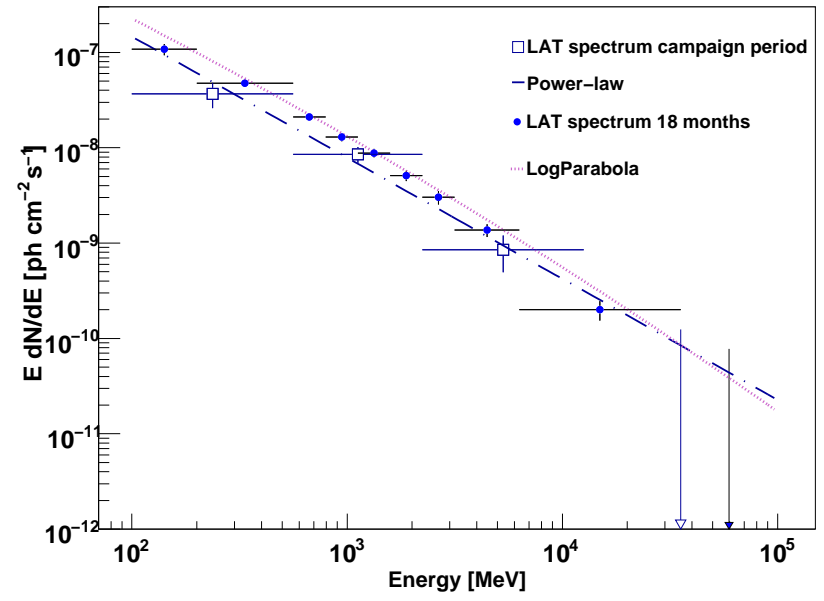

FIG. 3.- 18-month averaged energy spectrum of BL Lac obtained with a band likelihood over the accumulated observations from 2008 Aug. 04 to 2010 Feb. 04 (MJD 54682.7-55232.9). The spectrum shows a departure from a simple power law that can be fitted by a log parabola function. The blue (open square) symbols represent the spectrum corresponding only to the 48day period of the multifrequency campaign.

as in Abdo et al. (2010b),

$$
\mathcal{C}=\sum_{i} \frac{\left(F_{i}-F_{i}^{P L}\right)^{2}}{\sigma^{2}+\left(f_{i}^{r e l} F_{i}\right)^{2}},
$$


TABLE 1

BL LAC $\gamma$-RAY SPECTRUM OBTAINED WITH LIKELIHOOD ANALYSIS IN EACH ENERGY BAND.

\begin{tabular}{lll}
\hline \hline $\begin{array}{l}E_{\min }-E_{\max } \\
{[\mathrm{MeV}]}\end{array}$ & $\begin{array}{l}\gamma \text {-ray flux } \\
{\left[\times 10^{-8} \mathrm{ph} \mathrm{cm}^{-2} \mathrm{~s}^{-1}\right]}\end{array}$ & Syst. \\
\hline & 18 months \\
\hline $100-200$ & $7.62 \pm 1.03$ & $10 \%$ \\
$200-562$ & $5.14 \pm 0.27$ & $5 \%$ \\
$562-794$ & $0.73 \pm 0.06$ & $5 \%$ \\
$794-1122$ & $0.45 \pm 0.04$ & $5 \%$ \\
$1122-1585$ & $0.31 \pm 0.03$ & $10 \%$ \\
$1585-2239$ & $0.18 \pm 0.02$ & $10 \%$ \\
$2239-3162$ & $0.11 \pm 0.02$ & $10 \%$ \\
$3162-6310$ & $0.10 \pm 0.01$ & $15 \%$ \\
$6310-35481$ & $0.04 \pm 0.01$ & $15 \%$ \\
\hline & Aug $19-$ Sep 9 & $5 \%$ \\
\hline $100-562$ & $7.18 \pm 2.12$ & $10 \%$ \\
$562-2239$ & $1.28 \pm 0.24$ & $15 \%$ \\
$2239-12589$ & $0.17 \pm 0.07$ & \\
\hline \hline
\end{tabular}

where $i$ is the index for a particular energy bin, $F_{i}$ is the observed flux, $F_{i}^{P L}$ is the flux in the $i t h$ bin predicted by the global power-law fit, $\sigma$ is the error in $F_{i}$, and $f_{i}^{r e l}$ is the relative systematic uncertainty in that bin (reported in Table 1 ). The power-law fit has two parameters: the normalization and photon index. The curvature parameter is expected to follow a $\chi^{2}$ distribution with $9-2=7$ degrees of freedom (d.o.f.) if the power law hypothesis is true. With $99 \%$ confidence the spectral shape is significantly different from a power law when $\mathcal{C}>18.48$. For the accumulated 18-month data on BL Lac we found $\mathcal{C}=20.88(0.1<E<100 \mathrm{GeV})$, which implies a curved spectrum. The same procedure, when applied to the spectrum of the 48-day multifrequency campaign, resulted in $\mathcal{C}=2.83(0.1<E<100 \mathrm{GeV})$, implying there is no significant evidence for deviation from a simple power-law in this spectrum (Figures 3 and 10). This does not necessarily mean that there is no curvature during this 48 -day period, because it could be due to the lower emission state and accumulated statistics.

To further explore curvature, the 18-month spectrum was fit with a log-parabola function in the $0.1-300 \mathrm{GeV}$ energy band. This was performed in two runs: first a fit with $E_{\text {break }}$ left free, then another with $E_{\text {break }}$ frozen at the value found in the first run $(300 \mathrm{MeV})$ to improve the calculation of the other parameter values. The result of the latter fit gives

$$
\begin{aligned}
d N / d E & =(2.02 \pm 0.07) \times 10^{-10} \\
& \times(E / 300 \mathrm{MeV})^{-((2.23 \pm 0.05)+(0.07 \pm 0.02) \log (E / 300 \mathrm{MeV}))}
\end{aligned}
$$

with TS $=2384.1$. The higher TS value shows that the 18 months LAT spectrum does not follow a simple power law function, and the log-parabola model can describe this spectrum.

The weekly light curve in the $0.1-100 \mathrm{GeV}$ band for the 18 month period is presented in Figure 2 (upper panel). In order to quantify the variability, the variability index as defined in Abdo et al. (2010b), was computed from

$$
V=\sum_{i} w_{i}\left(F_{i}-F_{w t}\right)^{2}
$$

where

$$
w_{i}=\frac{1}{\sigma^{2}+\left(f^{r e l} F_{i}\right)^{2}},
$$

$$
F_{w t}=\frac{\sum w_{i} F_{i}}{\sum w_{i}}
$$

and the index $i$ runs over all data points except the upper limits (61 bins). Here $F_{i}$ and $\sigma_{i}$ are flux values and the relative statistical errors, respectively, and $f_{r e l}$ is equal to $3 \%$ of the flux for each interval, as suggested by Abdo et al. (2010b). For the 18 months, $F_{w t}$ was found to be $17.48 \times 10^{-8} \mathrm{ph}$ $\mathrm{cm}^{-2} \mathrm{~s}^{-1}$ and $\mathrm{V}=193$ with 60 d.o.f., which is consistent with variability (the probability the source is non-variable is less than $10^{-15}$ ). The same values computed just for the first 9 weeks, containing the period of the MW campaign, give $F_{w t}=10.67 \times 10^{-8} \mathrm{ph} \mathrm{cm}^{-2} \mathrm{~s}^{-1}$ and $V=0.6$ with 8 d.o.f., which is consistent with a non-variability hypothesis at $>99 \%$ confidence. Each of the LAT flux data points in the upper panel of Figure 4 has $T S>10$.

This interval with no observed $\gamma$-ray variability characterizes the intensive coordinated campaign period. After the campaign, starting from about halfway through 2008 November until 2010 February 4, BL Lac showed variable weekly $\gamma$-ray flux with $V=162$ with 51 d.o.f., $F_{w t}=19.15 \times 10^{-8}$ ph cm $\mathrm{cm}^{-2} \mathrm{~s}^{-1}$. In particular, a 1-week $\gamma$-ray flare occurred in the week MJD 54928.5-54935.5 (2009 Apr. 7-14), with a flux value of $72 \times 10^{-8} \mathrm{ph} \mathrm{cm}^{-2} \mathrm{~s}^{-1}$. This is about 4 times greater than the mean flux value $\left(F_{w t}\right)$ computed above. Moreover this is the highest weekly flux yet detected from BL Lac, although another likely flare was detected in the week around MJD 55221 (2010 Jan. 25, Sokolovsky et al. 2010a). After the 2009 April flare the source also showed slightly higher activity and flux.

The 78 points of the 18-month light curve were also used to compute the global normalized excess variance, as defined in Vaughan et al. (2003); Abdo et al. (2010d): $\sigma_{N X S}^{2}=$ $\left(S^{2}-\sigma_{e r r} \overline{2}\right) / \bar{x}^{2}$, where $S^{2}$ is the variance of the light curve and $\sigma_{\text {err }}^{2}=\sigma_{i}^{2}+\sigma_{\text {sys }}^{2}$, the sum in quadrature of the statistical uncertainty of the flux in the time bin and the systematic error estimate $\left(0.03\left\langle F_{i}\right\rangle\right)$. This quantity measured an intrinsic variability amplitude of $0.23 \pm 0.03$, in good agreement with the value found in Abdo et al. (2010d) reporting the weekly light curve of BL Lac (flux $E>300 \mathrm{MeV}$ ) during the first 11 months. This confirms again that BL Lac was variable after the first couple of months of Fermi science operations.

\section{OBSERVATIONS AND RESULTS FROM THE MULTIFREQUENCY CAMPAIGN}

The 48-day PIC on BL Lac involved the participation of the RXTE and Swift X-ray satellites, and of ground-based radio and optical observatories. These included the Metsähovi $13.7 \mathrm{~m}$ radio-telescope operating at $37 \mathrm{GHz}$ in Finland; the Very Long Baseline Array (VLBA) in the USA, which took a multi-waveband flux-structure observation on 2008 September 2; two telescopes of the Tuorla Observatory, Finland, and two telescopes of the Steward Observatory, USA, for the longterm and single-band optical monitoring as presented in Figure 2, and the $2.1 \mathrm{~m}$ optical telescope of the Observatorio Astrofísico Guillermo Haro (OAGH, Mexico) operating in nearinfrared, and the $1.3 \mathrm{~m}$ McGraw-Hill optical Telescope of the MDM Observatory (Arizona, USA), for further multi-band optical snapshots during the campaign period (Table 2).

\subsection{X-ray and $U V$ observations and results}

The RXTE/PCA space observatory performed sub-daily monitoring of BL Lac for 20 days, during the same epoch 


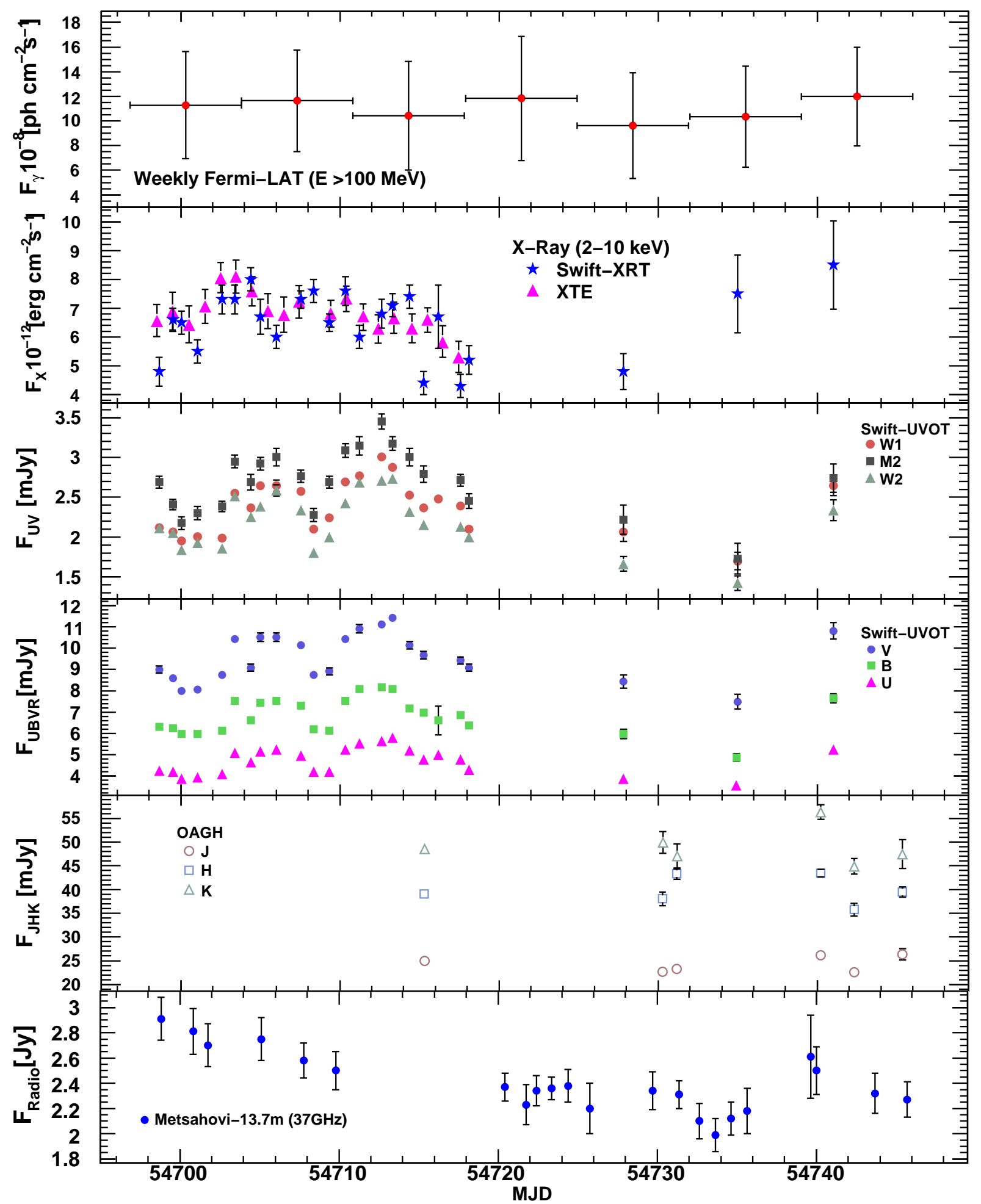

FIG. 4.- Simultaneous multifrequency flux light curves of BL Lac from the 48-days Fermi intensive campaign obtained with Fermi -LAT, Swift - XRT and UVOT, OAGH, and Metsähovi observations. LAT weekly fluxes reported in the upper panel for this period are all $T S>10$ detections. UV-to-near-IR fluxes are corrected for absorption.

as LAT and Swift, for a total of 80 pointings between 2008 August 20 and September 9. Only PCU2 was active, for a total exposure of $157.3 \mathrm{ks}$. The PCA STANDARD2 data were reduced and analyzed with the routines in HEASOFT V6.8 using the filtering criteria recommended by the RXTE Guest Observer Facility. Only the top-layer events were processed, and a check was performed in the $40-100 \mathrm{keV}$ range to assure that the model background reproduced the observed one. The average net count rate in the $3-18 \mathrm{keV}$ band is $0.55 \pm 0.01$ cts s$^{-1} \mathrm{pcu}^{-1}$. The RXTE spectra were extracted and fitted separately for each pointing, and summed to obtain the average spectrum. Each spectrum is well-fit by a single power- 
TABLE 2

BL LAC COORDINATED MULTIFREQUENCY CAMPAIGN (PIC) AND LONG-TERM MONITORING OBSERVATIONS

\begin{tabular}{lccc}
\hline \hline PIC (48 days) & & & \\
Instrument & Energy Range & 2008 Epoch range & \# obs. \\
\hline VLBA & $4.6-43.2 \mathrm{GHz}$ & Sep 2 & 7 \\
Metsähovi & $37 \mathrm{GHz}$ & Aug 20 - Oct 6 & 22 \\
OAGH & J H K & Sep 6 - Oct 6 & 18 \\
MDM & U B V R I & Oct 6 - 10 & 15 \\
Swift -UVOT & W2 M2 W1 U B V & Aug 20 - Oct 2 & 141 \\
Swift -XRT & $0.4-8 \mathrm{KeV}$ & Aug 20 - Sep 18 & 24 \\
RXTE -PCA & $3-18 \mathrm{KeV}$ & Aug 20 - Sep 8 & 19 \\
Fermi-LAT & $100 \mathrm{MeV}-100 \mathrm{GeV}$ & Aug 19- Oct 7 & 48 days \\
\hline \hline 18 months & & & \\
Instrument & Energy Range & Epoch range (MJD) & \# obs. \\
\hline Tuorla & $\mathrm{R}$ & $54709.8-55191.8$ & 162 \\
Steward & $\mathrm{V}$ & $54743.2-55213.1$ & 89 \\
Fermi-LAT & $100 \mathrm{MeV}-100 \mathrm{GeV}$ & $54682.7-55070$ & 78 weeks \\
\hline \hline
\end{tabular}

law model with Galactic absorption. The Galactic $N_{H}$ was fixed at two values: that measured by Elvis et al. (1989), based on dedicated 21-cm observations $\left(2.015 \times 10^{21} \mathrm{~cm}^{-2}\right)$; and the sum of this value with that inferred from millimeter observations (Lucas \& Liszt 1993), which includes the contribution from molecular hydrogen along the line of sight $\left(N_{\mathrm{H}} \simeq 3.6 \times 10^{21} \mathrm{~cm}^{-2}\right)$. In the RXTE band, however, this difference yields negligible effects, with a difference in spectral indices $\Delta \Gamma_{X} \sim 0.02$.

The RXTE data showed modest flux variations, with a rate which remained constant at $\sim 0.6 \mathrm{cts} \mathrm{s}^{-1}$ up to MJD 54708, and slowly decreasing to $\sim 0.4 \mathrm{cts} \mathrm{s}^{-1}$ towards MJD 54718 . Spectral variations were modest as well, ranging from $1.95 \pm$ 0.09 to $2.28 \pm 0.07$. The total average spectrum is well-fit with a single power-law model, with $\Gamma_{X}=2.18 \pm 0.06$, and an unabsorbed flux in the $2-10 \mathrm{keV}$ band of $F_{2-10}=(7.2 \pm$ $0.3) \times 10^{-12} \mathrm{erg} \mathrm{cm}^{-2} \mathrm{~s}^{-1}$ and $\chi_{r}^{2}=0.81$ (15 d.o.f.). This spectrum can also be fit by a concave log parabola or broken power-law model, with the slope hardening above $8-9 \mathrm{keV}$ from $\sim 2.2$ to $\sim 1.9\left(\chi_{r}^{2} \simeq 0.7\right)$; however, the low statistics do not allow one to determine if the improvement is significant, and the single, rather flat $\left(\Gamma_{X} \simeq 2\right.$, therefore $\left.\nu F_{\nu} \propto \nu^{0}\right)$, power law gives a good fit.

In the context of the overall SED, the low X-ray flux and the $\Gamma_{X} \simeq 2$ spectrum (flat X-ray SED) is evidence that the $R X T E$ band might correspond to the passage between the two humps of the blazars' SED, as typical for ISPs. In the following we show that the Swift -XRT results provide us further evidence for this conclusion.

The Swift gamma-ray burst satellite (Gehrels et al. 2004) performed a daily monitoring simultaneous to RXTE and LAT observations for 20 days (from 2008 Aug. 20, 15:19 UT, to 2008 Sep. 09, 02:37 UT; MJD 54698.64 - 54718.11), plus 3 more separated observations after 18 September. Data reduction and analysis was performed running a processing script customized for the XRT and UVOT data (Donato et al., in preparation). The script reprocesses the Swift data, stored in the HEASARC archive using the standard HEASoft software (version 6.8) and the latest calibration database (20091130 for XRT and 20100129 for UVOT). The reduction of XRT data consists of running xrtpipeline, selecting only the events with 0-12 grades in photon counting mode (PC). The UVOT image mode data are processed following the steps reported in the UVOT Software Guide 2.2.

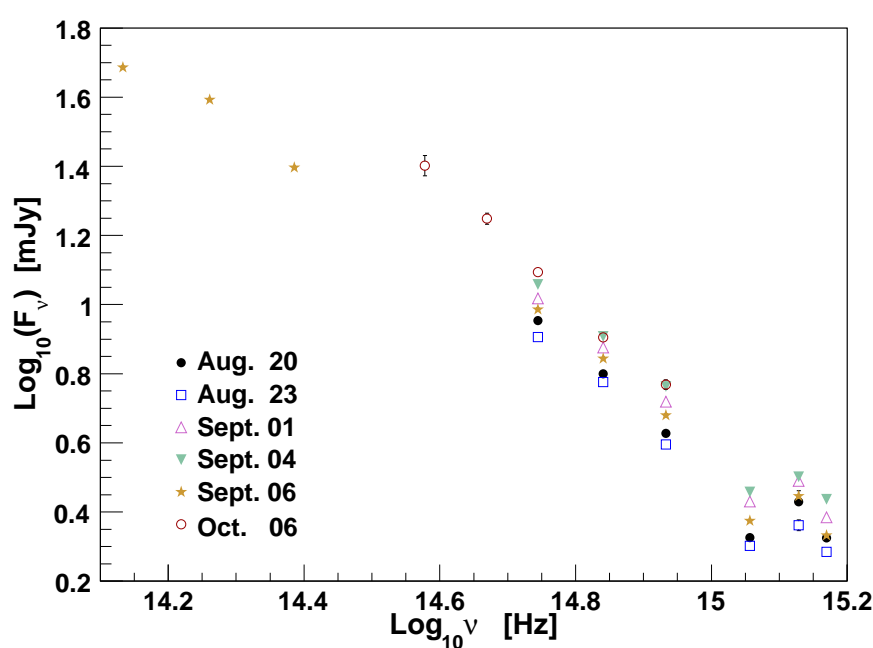

FIG. 5.- Simultaneous near-IR to UV SEDs of BL Lac at different epochs, collected with UVOT (Aug. 20, 23, Sept. 1, 4), OAGH (Sept. 6), and MDM (Oct. 6) observations.

The XRT data analysis is performed using the script xrtgrblc, available in the HEASoft software package. In brief, the script selects the best source and background extraction regions based on the source intensity (a circle and an annulus, respectively). In the case of BL Lac, the source extraction region has typically a $55^{\prime \prime}$ radius and the background region has a $110^{\prime \prime}-210^{\prime \prime}$ inner-outer radius. Field sources are excluded applying circular masks whose radius depends on the field source intensity. Using these regions, source and background count rates, spectra and event lists are extracted. The net source count rate must be corrected for irregularities in the exposure map and the Point-Spread Function (PSF). The total correction factor is obtained using the HEASoft tool xrtlccorr.

The script that handles the UVOT analysis is uvotgrblc (available within HEASoft). It determines the presence of field sources and excludes them from the background region with circular regions whose sizes depend on their intensity. The optimal background region is chosen after comparing 3 annular regions centered on the main source in the summed images. The region with the lowest background is selected. The inner and outer radii are $27^{\prime \prime}$ and $35^{\prime \prime}$, respectively, for all the filters except $V$, for which the two radii are $35^{\prime \prime}$ and $42^{\prime \prime}$. The source extraction region is also intensity dependent: The script selects the aperture size between $3^{\prime \prime}$ and $5^{\prime \prime}$ based on the observed source count-rate and the presence of close field sources. BL Lac is a relatively bright object and an extraction region of $5^{\prime \prime}$ is preferred. The field of view in the $V$ and $B$ filters is full of field sources and in particular there is a bright star east of the blazar. To avoid contamination from such a source, the extraction region is reduced to $3^{\prime \prime}$. The script estimates the best source position using the task uvotcentroid and the photometry using the task uvot source. The obtained values are already corrected for aperture effects while we used the dust maps of Schlegel et al. (1998) and the Milky Way extinction curve of Pei (1992) to compensate the Galactic extinction. XRT and UVOT light curves and SEDs are shown in Figures 4, 5, and 6 .

As with the harder energy band with RXTE, BL Lac does not show any major sign of variability in the Swift-XRT data. The same Galactic $\left(N_{\mathrm{H}} \simeq 3.6 \times 10^{21} \mathrm{~cm}^{-2}\right)$ fixed value was used also for the XRT analysis. The $2-10 \mathrm{keV}$ flux de- 

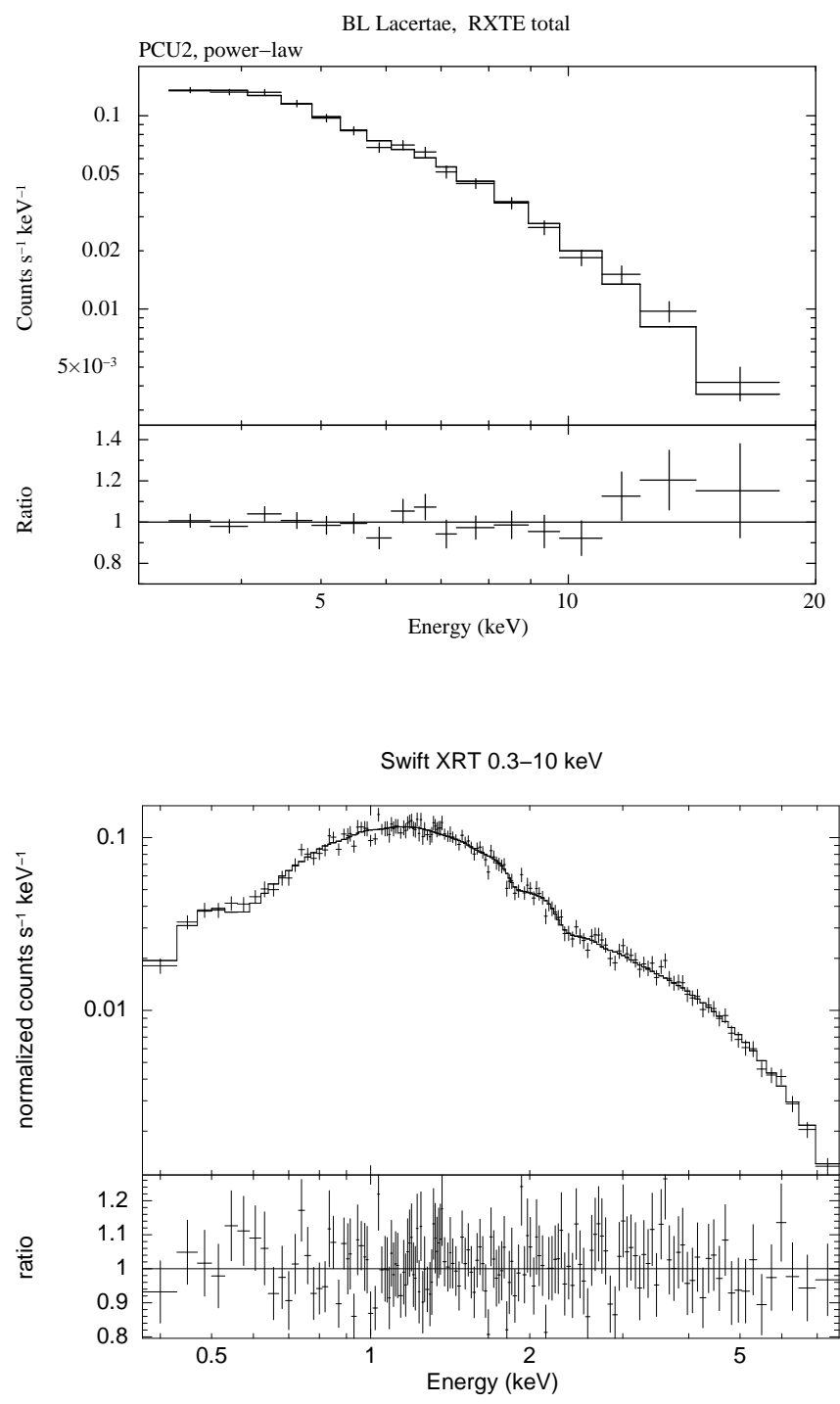

FIG. 6.- Top panel: RXTE combined 3-18 keV spectrum of BL Lac extracted cumulating the 20 days of observations of this X-ray satellite during the campaign. Bottom panel: Swift -XRT combined 0.4-8 keV spectra of BL Lac extracted cumulating the 24 days of observations of this X-ray satellite during the campaign.

rived from Swift observations lies between 6 and $8 \times 10^{-12}$ erg $\mathrm{cm}^{-2} \mathrm{~s}^{-1}$ with a hint of a lower intensity at the end of the campaign when the flux was below $5 \times 10^{-12} \mathrm{erg}$ $\mathrm{cm}^{-2} \mathrm{~s}^{-1}$. We found that in the day-by-day spectral analysis the photon index does not change significantly either. Using a single power-law model with fixed Galactic absorption, Leiden/Argentine/Bonn (LAB) Survey of Galactic HI Kalberla et al. 2005) weighted average equal to $1.73 \times 10^{21}$ $\mathrm{cm}^{-2}$ ), the slope varies between 1.75 and 2.06. Since the typical error on this parameter is of the order of 0.1 , the variability is within 2 sigma.

The absence of spectral changes allows us to extract an accumulated spectrum. As a first step, a comparison of the XRT and RXTE spectra accumulated over the same period and using the same lower energy threshold of $3 \mathrm{keV}$ results in very good agreement with the power-law photon index $\Gamma_{X}$ found by $R X T E$. The best fit in the $3-8 \mathrm{keV}$ range is obtained with a power-law whose slope is $2.19_{-0.11}^{+0.13}$. The $2-10 \mathrm{keV}$ integrated flux is $6.37_{-0.11}^{+0.12} \times 10^{-12} \mathrm{erg} \mathrm{cm}^{-2} \mathrm{~s}^{-1}$. The X-ray photon index is consistent within $1 \sigma$ with the results obtained using $R X T E$ data.

Using the entire XRT energy band from 0.3 to $10 \mathrm{keV}$ (Figure 6) and profiting from the increased statistics (the spectrum is binned with 100 counts/bin), the spectral fit is more constrained and underlies two emission components: the best fit ( $\chi_{r}^{2}=0.82$ with 146 d.o.f.) is obtained with a broken power-law with the energy break located at $1.71_{-0.14}^{+0.15} \mathrm{keV}$ and fixed absorption at the Galactic value. The soft and hard photon indices are $2.57_{-0.05}^{+0.06}$ and $2.09 \pm 0.05$, respectively and therefore the break in this XRT spectrum is significant compared to the power law. The $0.3-10 \mathrm{keV}$ integrated flux is $9.67_{-0.10}^{+0.14} \times 10^{-12} \mathrm{erg} \mathrm{cm}^{-2} \mathrm{~s}^{-1}$.

The UVOT optical-UV light curves showed some variability during the campaign period (Figure 4), but this is not well correlated with the X-ray or $\gamma$-ray emission (which is not variable). The daily simultaneous near-IR, optical, and UV SEDs, reconstructed thanks to UVOT, and OAGH observations (reported in Figure 5), showed no significant spectral variability and a trend consistent with a single power law except for the 2 higher frequency UV bands. This may be in agreement with the hypothesis of UV excess due to thermal emission from an accretion disk (Raiteri et al. 2009, 2010).

\subsection{Radio band flux-structure observations and results}

As part of an ongoing blazar monitoring program, the $37 \mathrm{GHz}$ observations were made with the $13.7 \mathrm{~m}$ diameter Metsähovi radio telescope, which is a radome enclosed paraboloid antenna situated in Finland. The measurements were made with a $1 \mathrm{GHz}$-band dual beam receiver centered at $36.8 \mathrm{GHz}$. The high electron mobility pseudomorphic transistor front end operates at room temperature. The observations are $\mathrm{ON}-\mathrm{ON}$, alternating the source and the sky in each feed horn. A typical integration time to obtain one flux density data point is $1200-1400 \mathrm{~s}$. The detection limit of the telescope at $37 \mathrm{GHz}$ is on the order of $0.2 \mathrm{Jy}$ under optimal conditions. Data points with a signal-to-noise ratio $<4$ are handled as non-detections. The flux density scale is set by observations of DR 21. Sources 3C 84 and 3C 274 are used as secondary calibrators. A detailed description on the data reduction and analysis is given in Teräsranta et al. (1998). The error estimate in the flux density includes the contribution from the measurement rms and the uncertainty of the absolute calibration.

BL Lac was also observed with the Very Long Baseline Array (VLBA) at seven frequencies $(4.6,5.0,8.1,8.4,15.4,23.8$ and $43.2 \mathrm{GHz}$ ) in the framework of a survey of parsec-scale radio spectra of twenty $\gamma$-ray bright blazars (Sokolovsky et al. $2010 \mathrm{~b})$. The multifrequency VLBA observation was carried out on 2008 September 2. The data reduction was conducted in the standard manner using the AIPS package (Greisen 1990). The final amplitude calibration accuracy is estimated to be $\sim 5 \%$ at 4.6-15.4 GHz range and $\sim 10 \%$ at 23.8 and 43.2 GHz. The Difmap software (Shepherd et al. 1994) was used for imaging and modeling of the visibility data.

The VLBA images (Fig. 7] and 8) reveal a wide, rather smooth, curved jet extending $\sim 50$ mas (at $5 \mathrm{GHz}$ ) south-east from the bright compact core. A few distinct, bright emission features aligned along the south-southwest direction can be seen in the inner jet at higher frequencies. We have modelled the observed brightness distribution with a small number of model components having two-dimensional Gaussian 


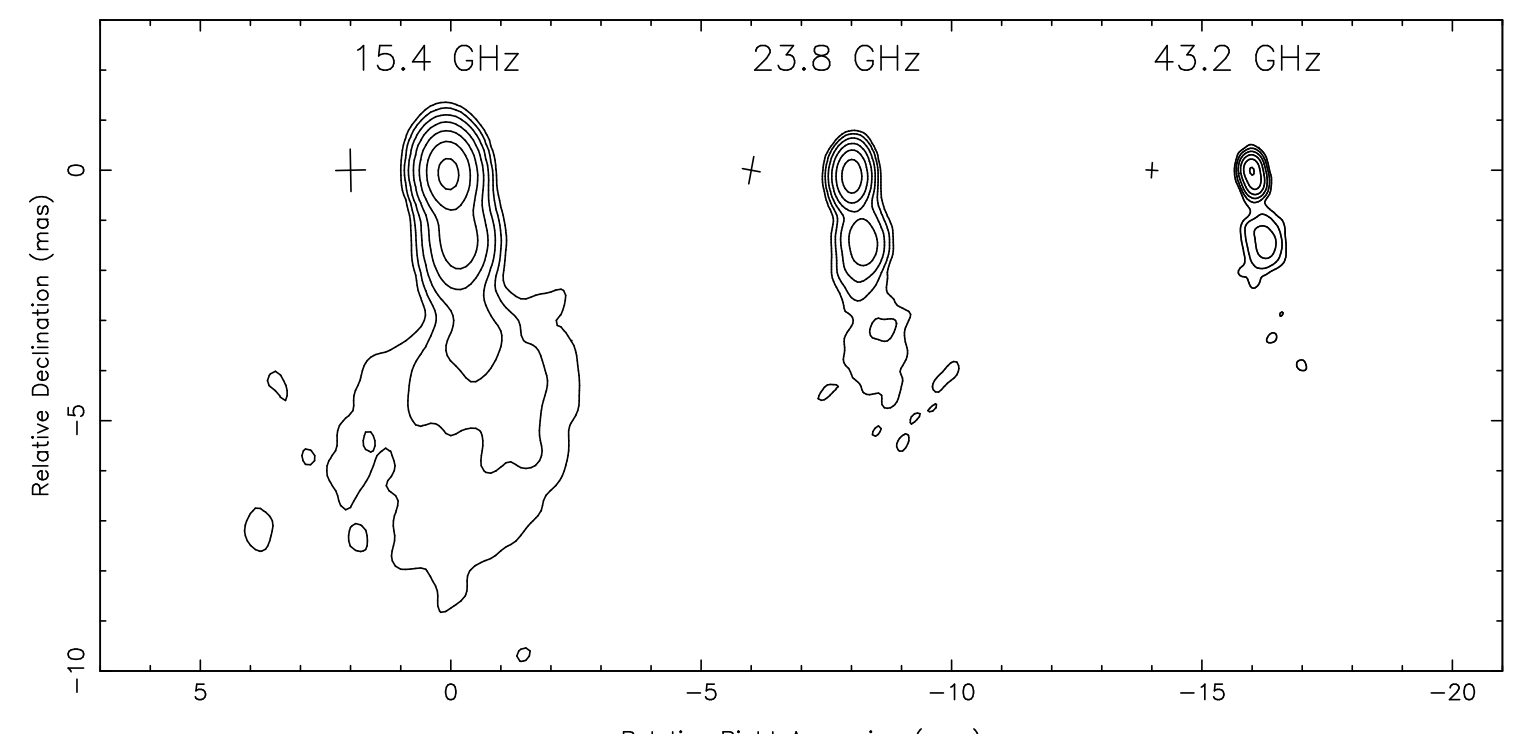

FIG. 7.- Inner jet of BL Lacertae as observed by the VLBA on September 02, 2008. Images at different frequencies are shifted by 8 mas in relative Right Ascension. For the 15.4, 23.8 and $43.2 \mathrm{GHz}$ image map peaks are 1.69, 1.52, 1.32 Jy/beam, first contours are 1.70, 3.00, 5.00 mJy/beam respectively. The contour levels are increased by a factor of 3 . Beam size (natural weighting) for each frequency is indicated by the cross to the left of the corresponding image.

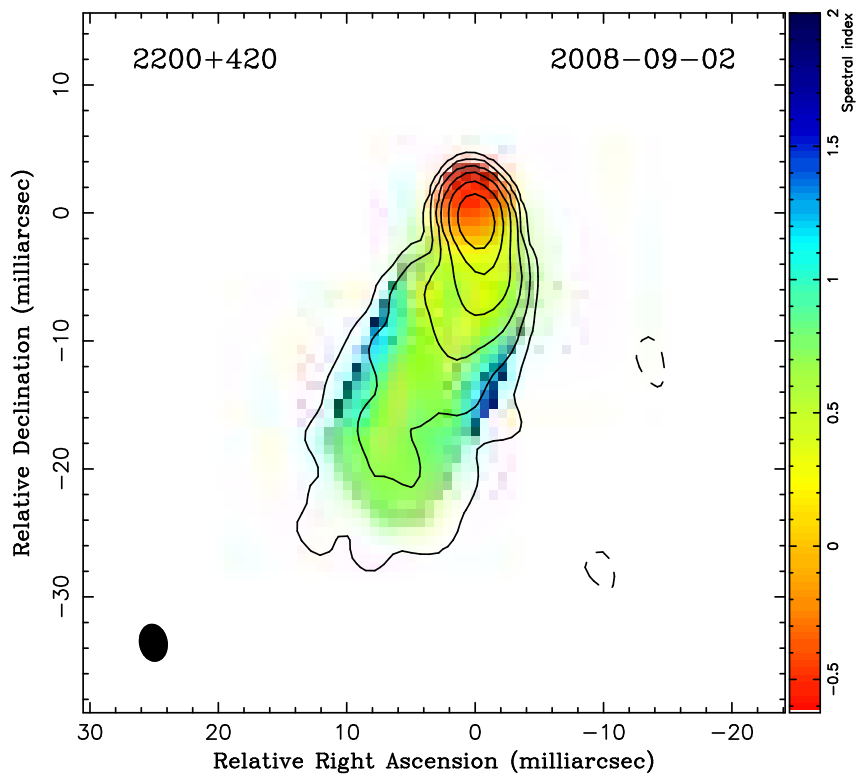

FIG. 8. - Spectral index map ( $F_{\nu} \sim \nu^{-\alpha}, \alpha$ is shown in color) of BL Lacertae constructed using VLBA observations at 4.6, 5.0, 8.1 and 8.4 GHz. The overlaid contours represent total intensity at $8.4 \mathrm{GHz}$ (the peak intensity is $1.75 \mathrm{Jy} / \mathrm{beam}$, the first contour is $0.70 \mathrm{mJy} / \mathrm{beam}$, the beam size is $1.57 \times 1.22$ mas at PA $10.4^{\circ}$.). The spectral index map was smoothed by a median filter with radius equal to the indicated beam size. The map shows an optically thin jet with $\alpha \sim 0.7$ and the self-absorbed core region $(\alpha<0.0)$. A 2D crosscorrelation technique using the optically thin part of the jet was employed to align images at different frequencies allowing reliable extraction of the spectral information. The spectral steepening towards the jet edges visible on the spectral index map occurs on the angular scale comparable to the beam size and is likely an effect of the uneven $u v$-coverage at different frequencies.

profiles. The core region (i.e., the bright feature at the north end of the jet) is elongated in the $43 \mathrm{GHz}$ image and can be modelled by two Gaussian components separated by 0.25 mas (0.32 pc projected distance). At a distances of 1.5 mas and 3.4 mas from the core, there are two other distinct emission features in the jet. A table listing the parameters of the distinct features ("components") can be found in Sokolovsky et al. (2010b).

The inner 0.25 mas part of the jet (i.e., the "core") may contain two (or more) distinct emission features or it may be a continuous emission region. The angular resolution even at $43 \mathrm{GHz}$ is not sufficient to distinguish between these possibilities. However, it is evident that the radio spectrum is changing along this region and it cannot be described by a single, uniform, self-absorbed, synchrotron emitting component. A turnover caused by the synchrotron self-absorption is detected in the averaged core spectrum at a frequency of $\sim 12 \mathrm{GHz}$ (an average over the whole area with the negative spectral index at Fig. 8). Due to the inhomogeneity, the innermost component at mm-wavelengths most likely has an even higher turnover frequency, $\geq 40 \mathrm{GHz}$. Using the method described in Sokolovsky et al. (2010b), the magnetic field, $B$, of the core, in the frame of the relativistic jet, can be constrained, given the Doppler factor, $\mathcal{D}=(\Gamma[1-\beta \cos \theta])^{-1}$, where $\Gamma=\left(1-\beta^{2}\right)^{-1 / 2}$ and $\theta$ here are the bulk Lorentz factor and jet angle to the line of sight, respectively. Assuming a Doppler factor $\mathcal{D}=7.3$ (Hovatta et al. 2009), an upper limit can be placed on the magnetic field strength in the core: $B<3 \mathrm{G}$. We note that this upper limit corresponds to a typical value across an extended and inhomogeneous region contributing the bulk of the radio emission in $4.6-43 \mathrm{GHz}$ range. The magnetic field strength can exceed the above limit locally or in a more compact regions hidden from sight in the observed frequency range by synchrotron opacity.

\subsection{Optical and near IR observations and results}

During the campaign several multi-band near-infrared and optical observations of BL Lac were also performed from the ground by the $2.1 \mathrm{~m}$ telescope of the OAGH observatory, Sonora, Mexico, and the $1.3 \mathrm{~m}$ McGraw-Hill Telescope of the MDM Observatory, Arizona, USA. In addition a longterm monitoring in optical $R$ and $V$ bands during the first 18 months of Fermi observations (Figure 2), was performed by the observing monitoring programs at the Tuorla Observatory ( $1 \mathrm{~m}$ Tuorla and $0.35 \mathrm{~m}$ KVA telescopes), Turku, Finland, and at the Steward Observatory (2.3 m Bok and $1.5 \mathrm{~m}$ Kuiper telescopes), USA respectively.

Optical $(U B V R I)$ data were taken with the $1.3 \mathrm{~m}$ McGraw-Hill Telescope (MDM observatory). Exposure times ranged from $40 \mathrm{~s}$ ( $R$ band) to $120 \mathrm{~s}$ ( $U$ band). The raw data 
were bias and flat-field corrected using IRAF. Instrumental magnitudes of BL Lac and comparison stars B, C, H, and K of Smith et al. (1985) were extracted using DAOPHOT and subsequently converted to physical magnitudes through differential photometry.

INAOE (Instituto Nacional de Astrofísica, Óptica y Electrónica) operates the Observatorio Astrofísico Guillermo Haro (OAGH) located in the Mexican state of Sonora. The $2.1 \mathrm{~m}$ telescope of the OAGH has a current allocation of over 50 nights per semester dedicated to the study of $\gamma$-ray sources, through optical photometry and spectroscopy and infrared photometry. The Cananea Near Infrared Camera, CANICA, is equipped with a Rockwell $1024 \times 1024$ pixel Hawaii infrared detector working at $75 \mathrm{~K}$ and standard $J, H$ and $K_{\mathrm{s}}$ bands. The plate scale in CANICA is 0.32 arcsec per pixel. Observations are usually carried out in series of 10 dithered frames in each filter. Data are co-added after bias and flatfield corrections. CANICA observations of BL Lac presented in this work were made on seven epochs between MJD 54715 and 54747. The data are shown in Figures 4 and 5, and do not show signs of variability.

The photometric and polarimetry monitoring program at the Tuorla Observatory, a division of the Department of Physics and Astronomy at the University of Turku, Finland, is performed through the $1.03 \mathrm{~m}$ telescope at Tuorla Observatory, and the $35 \mathrm{~cm}$ telescope at the KVA observatory on La Palma, Canary islands, Spain. Most of the monitored sources are BL Lac objects listed in Costamante \& Ghisellini (2002), as potential $\mathrm{TeV} \gamma$-ray sources. All photometric measures are taken using the R-filter (other details in Takalo et al.|2008).

The optical spectropolarimetry and spectrophotometry of blazars at the University of Arizona, is performed on a regular basis since the launch of Fermi using the Steward Observatory $2.3 \mathrm{~m}$ Bok telescope on Kitt Peak, Arizona, and $1.54 \mathrm{~m}$ Kuiper telescope on Mt. Bigelow, Arizona, USA. Depending on weather conditions, BL Lac is observed nightly during each of the monthly monitoring campaigns, which typically last a week. All of the measurements have been made with the SPOL spectropolarimeter (Schmidt et al. 1992). The Steward Observatory blazar monitoring program associated with the Fermi mission is described by Schmidt et al. (2009) and the data are publicly available 70 . Details concerning the reduction and calibration of both the polarimetry and photometry can also be found in Schmidt et al. (2009) and references therein. The data products include high signal-to-noise flux and linear polarization spectra spanning $\lambda=4000-7550 \AA$ and differential V-band photometry.

\subsection{Multifrequency correlation and SED}

The optical $R$ - and $V$-band light curve in the bottom panel of Figure 2 shows more rapid variability due to the increased sampling with respect to the weekly-averaged LAT light curve. Overlapping time intervals of the 18-month light curves in $\gamma$-rays and in the $R$ band were used to calculate the discrete cross correlation function (DCCF), following Edelson \& Krolik (1988). In Figure 9 the DCCF is plotted with error bars estimated by a Monte Carlo method, taking measurement errors and data sampling into account (Peterson et al. 1998). The correlation strength and lag were computed by fitting a gaussian profile to the DCCF between

\footnotetext{
69 http://users.utu.fi/kani/1m/

70 http://james.as.arizona.edu/ psmith/Fermi
}

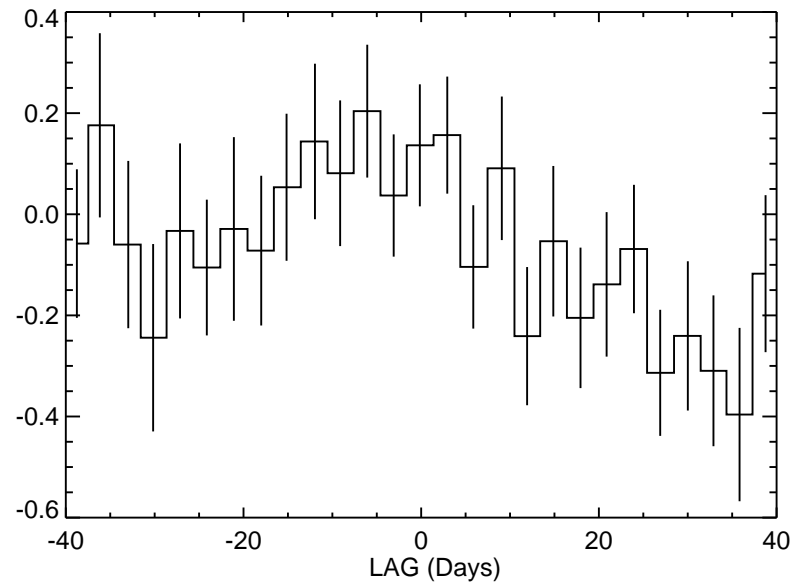

FIG. 9.- Discrete cross correlation function (DCCF) between the $\gamma$-ray and optical light curves of Fig. 2

lag -20 to +20 days. The result was a correlation intensity of $0.17 \pm 0.09$ (corrected for the effect of measurement noise) and a lag of $-5 \pm 5$ days, where negative lag means $\gamma$-rays lagging the $R$ band. The choice of lag range for the fit, from $-15,+15$ to $-30,+30$, as well as bin size, 3 or 4 days, only had a small effect on the estimated time lag of the peak and on the correlation strength, with the latter ranging from 0.14 to 0.17 . The rather weak long-term correlation between gamma-rays and optical tends to disfavor a one-zone synchrotron plus synchrotron self-Compton (SSC) model, since in this model one would expect electrons with approximately the same energies to make both the optical and $\gamma$-ray emission, and might favor an external radiation Compton (ERC) or multi-zone synchrotron/SSC model (e.g., Aharonian et al. 2009; Böttcher et al. 2009). The optical-UV variability observed during the 48 days of the multiwavelength campaign is not correlated with the weaker X-ray variability seen by Swift-XRT and RXTE nor with the non-variable $\gamma$-ray emission observed in the same period by the LAT (Figure 4). The lack of correlation might support the scenario where both synchrotron and IC photons contribute to the X-ray emission. Variability produced by the highest energy electrons would be smeared out by the IC component, and the mix of photons from the low and high energy component would dilute the correlation with the synchrotron, or synchrotron plus thermal, optical-UV emission. This would explain why variability in the optical/UV is greater than in X-rays. Moreover if the synchrotron and IC emission components come from physically distinct regions, they would also weaken the correlation. No evidence for a contribution by accretion disk radiation during this low activity state of the campaign was found, although this might contribute to the lack of clear correlation between the X-ray and optical-UV emission. The recent discovery of a luminous $H \alpha$ emission line in the optical spectra of BL Lac (Vermeulen et al. 1995; Corbett et al. 1996, 2000; Capetti et al. 2010), as likely a product of the broad line region photoionized by the disk radiation, can suggest a more relevant role played by the disk during major outburst with a high $\gamma$-ray Compton dominance (as seen in the EGRET 1997 outburst).

The averaged SED from the MW campaign(2008 Aug. 20 - Sep. 9) is reported in Figure 10. For the first time the multifrequency emission for the eponymous blazar source BL Lac is mapped from radio to $\mathrm{GeV} \gamma$-rays during a low-activity state. The relevant archival observations of BL Lac are also 


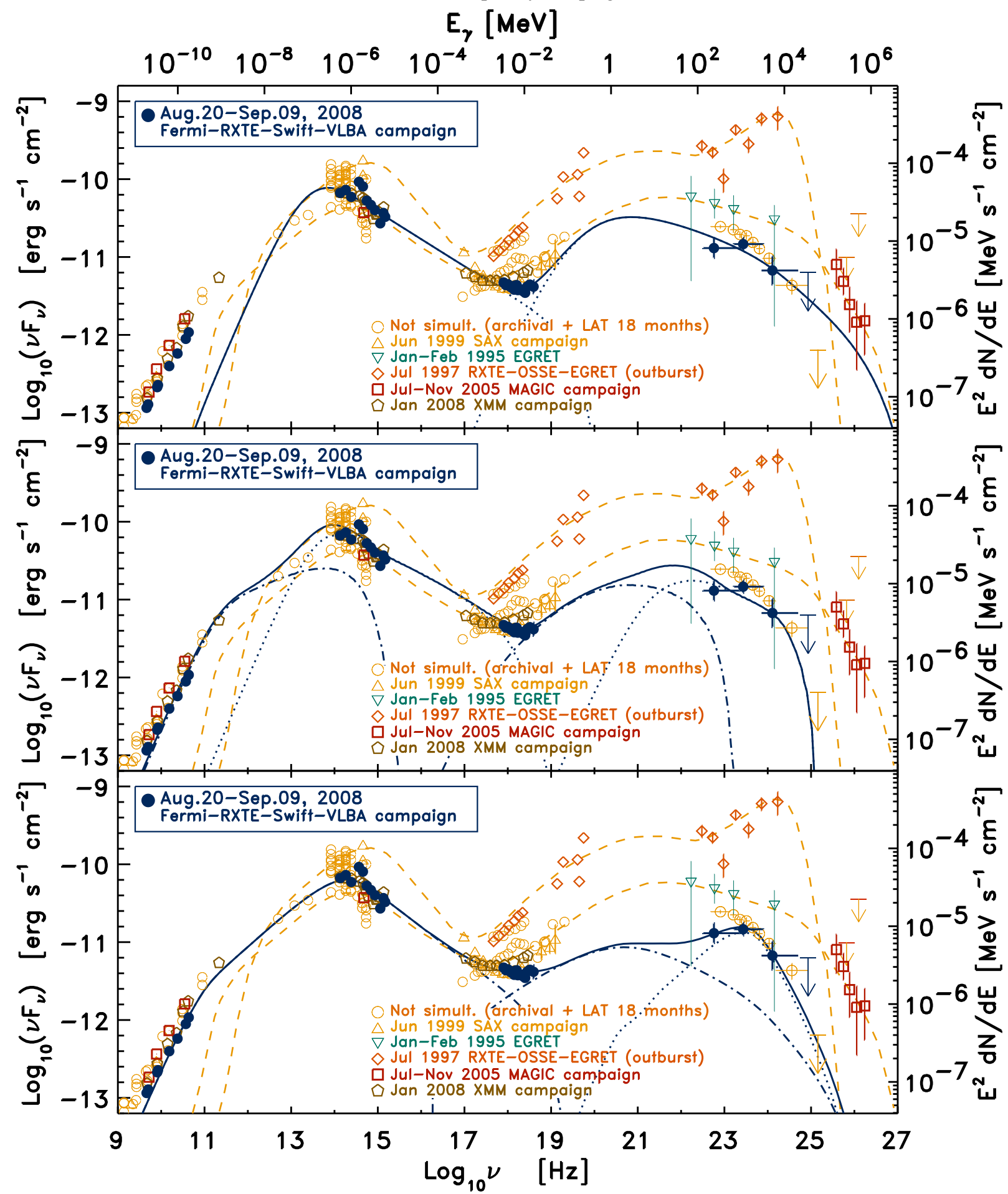

FIG. 10.- 2008, Aug. 20 - Sep. 09 simultaneous SED of BL Lac assembled with data of the coordinated campaign, and some archival data from the literature for comparison. The data are modeled with a single zone pure-SSC (top panel), two-zone pure SSC description (center panel), and a single zone SSC plus ERC descriptions (bottom panel). In the two-zone pure SSC description the blue/dark dotted line represents the compact, faster and high-energy emission component while the blue/dark dashed-dotted line is the larger and diluted zone emission component. In the SSC plus ERC model the blue/dark is the ERC emission (disk emission negligible here) component and the blue/dark dashed-dotted line is the synchrotron and SSC emission component. Past published and archival data are taken from Böttcher \& Bloom (2000); Ravasio et al. (2002); Böttcher et al. (2003); Albert et al. (2007); Raiteri et al. (2009), and fit models for two of these past epochs (the 1997 outburst campaign and the 2005 campaign, Ravasio et al. 2002; Albert et al. 2007) are represented as orange/clear long-dashed lines in each panel.

reported in this figure for comparison in addition to our data. The difference in $\gamma$-ray integrated flux with respect to the outburst state of 1997 measured by EGRET is about 15 times lower.

The XRT spectral results are in agreement with the
RXTE results in the common energy range $2-10 \mathrm{keV}$, and with the usual SED properties of ISP blazars. In fact the confirmation of a flat X-ray SED ( $\nu F_{\nu} \simeq$ const) of moderate flux level with both instruments in such range, makes us more confident the X-rays are from a transition region between the 
synchrotron and inverse Compton energy components. In addiction, as reported in Section 3.1 the whole 0.3-10 keV range spectrum accumulated during the campaign by Swift -XRT showed a broken power-law best fit, i.e. a steeper soft Xray spectral slope which should be the tail of the synchrotron emission and a flatter slope above $3 \mathrm{keV}$, which should be the first part of the IC energy component.

The LAT $\gamma$-ray spectrum accumulated in 18-months is reported as well for comparison (open/orange circles representing non simultaneous data in Fig. (10).

A single-zone synchrotron self-Compton (SSC) description, usually successful for $\mathrm{TeV}$ blazars, has been used to model also the SED of this eponymous BL Lac in the past (Ravasio et al. 2002, 2003), and is thus the first step in the attempt to reproduce its broadband emission. This model (Finke et al.2008) is applied to the SED of BL Lac (blue/dark thick line on the top panel of Figure 10). The energetic blob is modeled with Doppler factor $\mathcal{D}=\Gamma=26$, comoving radius $R=5.3 \times 10^{15} \mathrm{~cm}$, (which is consistent with a 2 hour variability time observed in the source on previous occasions), and tangled magnetic field of intensity $B=0.33 \mathrm{G}$. The electron distribution has the form of a broken power law, so that $N_{e}(\gamma) \propto \gamma^{-p_{1}}$ for $\gamma_{\text {min }}=600<\gamma<\gamma_{\text {break }}=1.4 \times 10^{3}$, and $N(\gamma) \propto \gamma^{-p_{2}}$ for $\gamma_{\text {break }}<\gamma<\gamma_{\max }=10^{6}$ where $\gamma=E /\left(m c^{2}\right)$ is the electron Lorentz factor. The best fit electron indices were found to be $p_{1}=2.0$ and $p_{2}=3.8$, which is consistent with electrons in the fast cooling regime. It is interesting to note that $\gamma_{\min }$ is well constrained, for a given a blob size, by the X-ray observations, and thus it is impossible for this model to reproduce the radio points. This means that, if this model is correct, the emitting region must be closer to the black hole than the radio photosphere. In this model the Poynting flux power and the kinetic power in relativistic electrons are $L_{j e t}^{(B)}=1.5 \times 10^{43} \mathrm{erg} \mathrm{s}^{-1}$ and $L_{\text {jet }}^{(e)}=2.2 \times 10^{44} \mathrm{erg} \mathrm{s}^{-1}$, respectively, implying an equipartition fraction $\epsilon_{B} \equiv U_{B}^{\prime} / U_{e}^{\prime}=L_{j e t}^{(B)} / L_{j e t}^{(e)}=0.068$. This is well below the equipartition value, which may be argued as a reason to disfavor this model. In this case the Doppler factor is considerably higher than the radio-variability Doppler factor reported in Hovatta et al. (2009), although they evaluated that from the estimated timescale of each radio flare component and the known brightness temperature, referred therefore to a much larger region beyond the radio photosphere than this model suggests.

Since the one-zone SSC model has difficulty reproducing the variability of this source and giving reasonable energetics $\left(\epsilon_{B}\right.$ substantially below 1$)$, as further step we attempt a fit with a two-zone SSC model (blue/dark thick line in the central panel of Figure 10, , using two emitting blobs via SSC emission: a compact and faster emission region and a larger, slower and diluted region accounting also for the radio-band emission. The energetic blob responsible for most of the emitted power and originating in a limited part of the jet is modeled with $\mathcal{D}_{1}=10, R_{1}=9.0 \times 10^{15} \mathrm{~cm}, B_{1}=0.45 \mathrm{G}$. The radio-band and hard $\mathrm{X}$-ray emission is reproduced using a much larger emitting region characterized by parameter values $\mathcal{D}_{2}=6.5, R_{2}=1.6 \times 10^{17} \mathrm{~cm}$, and $B_{2}=0.02$ G. Doppler factors of the two zones are found to be closer to the average value $(\mathcal{D}=7.3)$ found in previous years by Hovatta et al. (2009) than the one-zone SSC model presented above. The kinetic partial differential equation of this model describes the evolution of the particle energy distribu- tion $N(\gamma, t)$ after the injection of shock-accelerated electrons, with a rate equal to a power law with an exponential cutoff: $Q(\gamma) \propto \gamma^{-p} e^{-\gamma / \gamma_{\max }},\left[\mathrm{cm}^{-3} \mathrm{~s}^{-1}\right]$, where $\gamma=E /\left(m c^{2}\right)$, between $\gamma_{\min }$ and $\gamma_{\max }$. More details on the numerical model can be found in (Ciprini 2008, 2010). For the compact region (1) we obtain $\gamma_{\min }^{(1)}=3.0 \times 10^{3}$ and $\gamma_{\max }^{(1)}=7.0 \times 10^{5}$ with soft injection index $p^{(1)}=3.5$, and $L_{\text {jet }(1)}^{(B)}=6.1 \times 10^{42}$ $\mathrm{erg} \mathrm{s}^{-1}, L_{j e t(1)}^{(e)}=2.2 \times 10^{42} \mathrm{erg} \mathrm{s}^{-1}$, implying an equipartition fraction $\epsilon_{B}^{(1)}=2.8$. Considering the larger diluted region (2) we obtain $\gamma_{\min }^{(2)}=600$ and $\gamma_{\max }^{(2)}=3.0 \times, 10^{4}$ with injection index $p^{(2)}=2.4$, and $L_{\text {jet }(2)}^{(B)}=1.6 \times 10^{42} \mathrm{erg} \mathrm{s}^{-1}$, $L_{\text {jet }(2)}^{(e)}=7.9 \times 10^{43} \mathrm{erg} \mathrm{s}^{-1}$, implying an equipartition fraction $\epsilon_{B}^{(2)}=0.020$. In this case for the two regions the total $\epsilon_{B}=2.16$, a value slightly closer to energy equipartition than the one-zone model. The hypothesis of two synchrotron components present at different distances from the nucleus of BL Lac and accounting for the optical and the X-ray spectra respectively, was suggested in the past in Ravasio et al. (2003). In our case X-rays could be considered produced from both the emitting regions (central panel of Figure 10), and this can explain the lack of evident correlation between UVOT and XRT/RXTE light curves. On the other hand the fact that the variations in $\gamma$-rays are not seen, or not resolved by the LAT, contrary to the optical-UV variable flux, tell us that the two-zone pure SSC model does not explain the multifrequency light curve of Figure 4 observed in BL Lac during the campaign, although the energetics is closer to (and above) equipartition with respect to the previous single-zone model.

SSC emission with the relevant addition of external radiation Compton (ERC) emission was used to explain the large Compton dominance of BL Lac during the 1997 $\gamma$-ray outburst recorded by EGRET (Bloom et al. 1997; Madejski et al. 1999; Böttcher \& Bloom 2000; Ravasio et al. 2002; Böttcher \& Reimer 2004). An accretion disk emitting component, and emission lines were also observed several times in this blazar (Vermeulen et al. 1995; Corbett et al. 1996, 2000; Capetti et al. 2010). The SSC plus ERC hybrid model, where X-ray emission is produced via the SSC mechanism and the GeV $\gamma$-ray emission is produced by ERC, was used by Madeiski et al. (1999) for this source. This motivates its use to see if it is able to reproduce also the 2008 low state of BL Lac. A model fit to this SED is shown as the blue/dark thick line of the bottom panel of Figure 10 . For this model fit an equilibrium version of the time-dependent jet model reported in Böttcher \& Chiang (2002) was used. A power-law distribution of electrons, $Q(\gamma)=Q_{0} \gamma^{-p}$, with lower and upper cut-off $\gamma_{\min }=1.1 \times 10^{3}$ and $\gamma_{\max }=2.0 \times 10^{5}$, respectively, and injection index $p=2.85$ is injected into a compact region of radius $R=3 \times 10^{15} \mathrm{~cm}$, moving relativistically along the jet, oriented at an angle $\theta_{\text {obs }}=3.8^{\circ}$ with respect to the line of sight, with bulk Doppler factor $\mathcal{D}=15$ and tangled magnetic field intensity $B=2.5 \mathrm{G}$. The instantaneous electron injection is self-consistently balanced with particle escape on a time scale $t_{\mathrm{esc}}=\eta_{\mathrm{esc}} R / c$, where $\eta_{\mathrm{esc}}=60$ is a free parameter, and radiative cooling through synchrotron, SSC and ERC emission. For the ERC emission, both the direct accretion disk radiation field and accretion disk emission reprocessed in the BLR are taken into account, although for our fit, the BLR-reprocessed radiation strongly dominates the radiation which is directly from the disk. We assume an ac- 
cretion disk luminosity of $L_{d i s k}=6 \times 10^{44} \mathrm{erg} \mathrm{s}^{-1}$, and an emitting region distance from the black hole of $r_{0}=0.1 \mathrm{pc}$. The BLR is represented as a spherical shell of reprocessing material with radial Thomson depth $\tau_{\mathrm{BLR}}=0.01$, located at a distance $r_{\mathrm{BLR}}=0.21 \mathrm{pc}$ from the central black hole. Although the model fits the radio data well, the assumed distance from the black hole within the BLR implies that the blazar region is well within the radio-band photosphere. The numerical model self-consistently evaluates the energy content in the resulting equilibrium electron distribution, and compares this value to the magnetic-field energy density. This model gives $L_{\text {jet }}^{(B)}=4.75 \times 10^{43} \mathrm{erg} \mathrm{s}^{-1}$ and $L_{\text {jet }}^{(e)}=3.2 \times 10^{43}$, and ratio $\epsilon_{B}=1.48$. This is closer to equipartition than the one- and two-zone pure SSC models described above. Considering the equipartition ratio $\epsilon_{B}$ the differences between the two-zones SSC and the ERC scenario do not appear particularly significant because of the uncertainties in the estimate of this parameter, but the uncorrelated variability can still be explained more easily in the ERC scenario.

The parameters used for the three SED models that are shown in Figure 10 are in agreement with the limit on the magnetic field obtained from the VLBA observations, while the Doppler factors used in the two-zone SSC are in better agreement with the value extracted from radio-band structure historical observations. The magnetic field and Doppler factor could be different for the $\gamma$-ray emitting region than for the radio core region described in section 3.2 , since the $\gamma$-ray emittion region could be from a smaller region than the radio core, closer to the black hole. A ERC+SSC model can produce more complex SEDs and less correlated variability patterns, partly because of variability in the external radiation field. The emission region could move through an inhomogeneous radiation field, when it leaves the BLR. Synchrotron and SSC depend on the magnetic field, while the ERC component does not. Therefore, magnetic field fluctuations may also produce uncorrelated variability patterns between optical - X-rays (SSC) on the one hand and $\gamma$-rays (ERC) on the other.

\section{CONCLUSIONS}

This is the first broad-band multifrequency campaign investigating the low activity state of BL Lac. This eponymous source is also peculiar, sometimes showing a rather complex SED profile, a behavior similar to an FSRQ (Vermeulen et al. 1995; Madejski et al. 1999), and being detected also by VHE Cherenkov telescopes like MAGIC above $200 \mathrm{GeV}$. We observed a $\gamma$-ray flux $(E>100 \mathrm{MeV})$ during the 48-day multifrequency campaign (planned intensive campaign, PIC) of 2008 about 15 times lower than the level measured by EGRET during the 1997 outburst, and lower than the 1995 EGRET observations. This value and the weekly light curve of this campaign interval indicates a quiescent $\gamma$-ray state. The average LAT spectrum accumulated during the first 18 months of survey showed evidence for curvature. The single photon of maximum energy detected by the LAT during these months was measured at $70 \mathrm{GeV}$, with a spectrum that could extend into the VHE range. The weekly light curve is characterized by at least two mild flares on the 1-week timescale (in the middle of 2009 April and the end of 2010 January).

During the PIC the low luminosity and non-variable $\gamma$-ray state did not correspond to the lowest luminosity state for near-IR and optical emission. No evidence for clear correlation between the low-energy emission component (radio to
UV bands) and the high-energy emission component (X-ray to $\gamma$-ray bands) was found. Since one would expect correlated variability between these components in a one-zone SSC scenario, a fit with a two-zone SSC model was attempted. This model as well is not able to explain the variable optical and UV emission seen by Swift corresponding to the non-variable $\gamma$-ray emission during the coordinated campaign. Therefore a hybrid SSC+ERC model was better able to reproduce the SED being closer to energy equipartition and providing a possible explanation of the uncorrelated variability.

This result tells us that the SSC+ERC model is valid for BL Lac also during low brightness states (strengthening the quasar-like, FSRQ, view of this blazar). The two-zone SSC model might still be a possibility for this blazar (as suggested, for example in Ravasio et al. 2003). Other blazars like S5 0716+71 appear to have evidence for two synchrotron components (Giommi et al. 2008; Chen et al. 2008). In 3C 454.3, the prototypical FSRQ, a two-zone synchrotron scenario is inferred by the variability of the dust IR emission bump resolved by Spitzer (Ogle et al. 2010). Therefore, as suggested in the past, BL Lac, a rather special object with a possible Seyfert-like nucleus, might be considered a miniature and real FSRQ (Vermeulen et al. 1995; Corbett et al. 1996; Madejski et al. 1999; Corbett et al. 2000; Capetti et al. 2010).

A multi-frequency VLBA observation performed during this campaign demonstrated that the bright, innermost part of the radio-mm jet ("the core") cannot be described by a single, uniform, self-absorbed, synchrotron emitting blob, but rather an inhomogeneous region with spatially changing spectral properties. The synchrotron self-absorption turnover is detected in the VLBA data which allows us to constrain the average magnetic field in the core region to be less than $3 \mathrm{G}$, assuming a particular Doppler factor from previous radio singledish observations. SED model values are in agreement with this limit, although this is not strictly necessary since the models assume emission from a region closer to the black hole than the radio core.

Another interesting discovery was that during the campaign, BL Lac continued to show a broken, concave X-ray spectrum, as seen by the Swift -XRT. This was not confirmed by $R X T E$, probably because of the differing energy range and worse sensitivity of this instrument, but the photon in$\operatorname{dex} \Gamma_{X} \sim 2$ is in agreement with this view. We note that the RXTE spectrum was mostly consistent with the XRT one. Both instruments observed an X-ray spectrum with small dayto-day X-ray photon index variations during this low-activity state. The concave spectrum and lack of spectral index variation is believed to be a typical signature of intermediate energy peaked blazars, and we find it exists even during nonflaring states.

\section{ACKNOWLEDGMENTS}

The Fermi -LAT Collaboration acknowledges generous ongoing support from a number of agencies and institutes that have supported both the development and the operation of the LAT as well as scientific data analysis. These include the National Aeronautics and Space Administration and the Department of Energy in the United States, the Commissariat à l'Energie Atomique and the Centre National de la Recherche Scientifique / Institut National de Physique Nucléaire et de Physique des Particules in France, the Agenzia Spaziale Italiana and the Istituto Nazionale di Fisica Nucleare in Italy, the Ministry of Education, Culture, Sports, Science and Technology (MEXT), High Energy Accelerator Research Organization (KEK) and Japan Aerospace Exploration Agency (JAXA) in Japan, and the K. A. Wallenberg Foundation, the Swedish Research Council and the Swedish National Space Board in Sweden.

Additional support for science analysis during the operations phase is gratefully acknowledged from the Istituto Nazionale di Astrofisica in Italy 
and the Centre National d'Études Spatiales in France.

S.C. acknowledges funding by grant ASI-INAF n.I/047/8/0 related to Fermi on-orbit activities.

This work includes observations obtained with the NASA Swift gammaray burst Explorer. Swift is a MIDEX Gamma Ray Burst mission led by NASA with participation of Italy and the UK. This work includes observations obtained with NASA Rossi XTE satellite. The ASM/RXTE teams at MIT and at the RXTE Science Operation Facility and Guest Observer Facility at NASA's GSFC are gratefully thanked. This work includes observations obtained with the Very Long Baseline Array, USA (project code BK150). The National Radio Astronomy Observatory (NRAO VLBA) is a facility of the National Science Foundation operated under cooperative agreement by Associated Universities, Inc. This work includes observations obtained with the $14 \mathrm{~m}$ Metsähovi Radio Observatory, a separate research institute of the Helsinki University of Technology. The Metsähovi team acknowledges the support from the Academy of Finland to our observing projects (numbers 212656, 210338, and others). This work includes observations ob- tained through the Tuorla Blazar Monitoring Program, carried out with the KVA telescope on La Palma, Canary Islands and the $1 \mathrm{~m}$ telescope at Tuorla. Tuorla Observatory is a division of the Department of Physics and Astronomy at the University of Turku, Finland. This work includes observations obtained through the optical monitoring of BL Lac and other blazars using the $2.3 \mathrm{~m}$ Bok and $1.54 \mathrm{~m}$ Kuiper telescopes of Steward Observatory that is supported by NASA/Fermi Guest Investigator grants NNX08AW56G and NNX09AU10G. This work includes observations obtained with the $2.1 \mathrm{~m}$ telescope of the OAGH Observatorio Astrofísico Guillermo Haro, in the state of Sonora, Mexico, operated by the Instituto Nacional de Astrofísica, Óptica y Electrónica (INAOE), Mexico. This work includes observations obtained with the $1.3 \mathrm{~m}$ McGraw-Hill Telescope of the Michigan-Dartmouth-MIT (MDM) observatory, operated by University of Michigan, Dartmouth College, Ohio State University, Ohio University, Columbia University, in Arizona, USA.

The Fermi LAT Collaboration extend thanks to the anonymous referee who made useful comments.

Facilities: Fermi, VLBA, RXTE, Swift

\section{REFERENCES}

Abdo, A. A., et al. 2010a, ApJ, 716, 30

Abdo, A. A., et al. 2010b, ApJS, 188, 405 ApJ, 710, 1271

Abdo, A. A., et al. 2010d, ApJ, 722, 520 2009, ApJ, 707, 612

Aharonian, F., et al. 2009, ApJ, 696, L150

Atwood, W. B., et al., 2009, ApJ, 697, 1071

Albert, J., et al. 2007, ApJ, 666, L17

Bach, U., et al. 2006, A\&A, 456, 105

Bloom, S. D., et al., 1997, ApJ, 490, L145

Böttcher, M., \& Bloom, S. D. 2000, AJ, 119, 469

Böttcher, M., \& Chiang, J., 2002, ApJ, 581, 127

Böttcher, M., et al. 2003, ApJ, 596, 847

Böttcher, M., \& Reimer, A. 2004, ApJ, 609, 576

Böttcher, M., Reimer, A., \& Marscher, A. P. 2009, ApJ, 703, 1168

Capetti, A., Raiteri, C. M., \& Buttiglione, S. 2010, A\&A, 516, A59

Catanese, M., et al. 1997, ApJ, 480, 562

Chen, A. W., et al. 2008, A\&A, 489, L37

Ciprini, S. 2008, in proc. of Blazar Variability across the Electromagnetic Spectrum, PoS(BLAZARS2008), n.073

Ciprini, S. 2010, Mem. Soc. Astronom. It. Supplements, 14, 262

Costamante, L., \& Ghisellini, G. 2002, A\&A, 384, 56

Corbett, E. A. et al. 1996, MNRAS, 281, 737

Corbett, E. A., Robinson, A., Axon, D. J., \& Hough, J. H., 2000, MNRAS, 311,485

Denn, G. R., Mutel, L. R., \& Marscher, A. P., 2000, ApJS, 129, 61

Edelson, R. A., \& Krolik, J. H. 1988, ApJ, 333, 646

Elvis, M., Wilkes, B. J., \& Lockman, F. J. 1989, AJ, 97, 777

Finke, J. D., Dermer, C. D., Böttcher, M. 2008, ApJ, 686, 181

Fossati, G., Maraschi, L., Celotti, A., Comastri, A., \& Ghisellini, G., 1998, MNRAS, 299, 433

Gehrels, N., et al. 2004, ApJ, 611, 1005

Giebels, B., \& Degrange, B. 2009, A\&A, 503, 797

Giommi, P., et al. 2008, A\&A, 487, L49

Greisen, E. W. 1990, Acquisition, Processing and Archiving of Astronomical Images, 125

Lucas, R., \& Liszt, H. S. 1993, A\&A, 276, L33

Hovatta T., Valtaoja E., Tornikoski M., Lähteenmäki A., 2009, A\&A, 494, 527

Kalberla, P. M. W., Burton, W. B., Hartmann, D., Arnal, E. M., Bajaja, E., Morras, R., Pöppel, W. G. L. 2005, A\&A, 440, 775

Komatsu, E., Dunkley, J., Nolta, M. R., et al. 2009, ApJS, 180, 330

Kranich, D. 2003, ASP Conf. Proc. 299, High Energy Blazar Astronomy, 3

Lister, M. L., Cohen, M. H., Homan, D. C., Kadler, M., Kellermann, K. I., Kovalev, Y. Y., Ros, E., Savolainen, T., Zensus, J. A. 2009, AJ, 138, 1874

Madejski, G. M., Sikora, M., Jaffe, T., Błażejowski, M., Jahoda, K., \& Moderski, R. 1999, ApJ, 521, 145
Marscher, A. P., et al. 2008, Nature, 452, 966

Mattox, J. R., Bertsch, D. L., Chiang, J., et al. 1996, ApJ, 461, 396

Nandikotkur, G., Jahoda, K. M., Hartman, R. C., Mukherjee, R., Sreekumar,

P., Böttcher, M., Sambruna, R. M., \& Swank, J. H. 2007, ApJ, 657, 706

Nesci, R., Maesano, M., Massaro, E., Montagni, F., Tosti, G., \& Fiorucci, M 1998, A\&A, 332, L1

Neshpor, Y. I., Chalenko, N. N., Stepanian, A. A., Kalekin, O. R., Jogolev,

N. A., Fomin, V. P., \& Shitov, V. G. 2001, Astronomy Reports, 45, 249

Ogle, P. M., Wehrle, A. E., Balonek, T., \& Gurwell, M. A. 2010, ApJS, submitted (arXiv: 1003.3642)

Padovani, P., \& Giommi, P. 1995, ApJ, 444, 567

Pei, Y. C. 1992, ApJ, 395, 130

Peterson, B. M., Wanders, I., Horne, K., Collier, S., Alexander, T., Kaspi, S., \& Maoz, D. 1998, PASP, 110, 660

Raiteri, C. M., et al. 2009, A\&A, 507, 769

Raiteri, C. M., et al. 2010, A\&A, 524, A43

Ravasio, M., et al. 2002, A\&A, 383, 763

Ravasio, M., Tagliaferri, G., Ghisellini, G., Tavecchio, F., Böttcher, M., \&

Sikora, M. 2003, A\&A, 408, 479

Sambruna, R., et al., 1999, ApJ, 515, 140

Schlegel, D. J., Finkbeiner, D. P., \& Davis, M. 1998, ApJS, 500, 525

Schmidt, G. D., Stockman, H. S., \& Smith, P. S. 1992, ApJ, 398, L57

Smith, P.S., Montiel, E., Rightley, S., Turner, J., Schmidt, G.D., \& Jannuzi,

B.T. 2009, 2009 Fermi Symposium, eConf Proceedings C091122, (arXiv:0912.3621)

Shepherd M.C., Pearson T.J., \& Taylor G.B., 1994, BAAS, 26, 987

Smith, P. S., Balonek, T. J., Heckert, P. A., Elston, R., \& Schmidt, G. D. 1985, AJ, 90, 1184

Sokolovsky, K. V., Schinzel, F. K., \& Wallace, E. 2010a, The Astronomer's Telegram, 2402,

Sokolovsky, K. V., Kovalev, Y. Y., Lobanov, A. P., Savolainen, T., Pushkarev, A. B., \& Kadler, M. 2010b, arXiv:1001.2591

Takalo, L. O., Nilsson, K., Lindfors, E., Sillanpää, A., Berdyugin, A., \& Pasanen, M. 2008, AIP Conference Series, 1085, 705

Teräsranta, H., Tornikoski, M., Mujunen, A., et al. 1998, A\&AS, 132, 305

Thompson, D. J. 2007, AIP Conf. Proc., 921, 86

Tosti, G., Luciani, M., Fiorucci, M. et al. 1999, Mem. Soc. Astron. Italiana, 70,237

Tosti, G. 2007, AIP Conf. Proc., 921, 255

Vaughan, S., Edelson, R., Warwick, R. S., \& Uttley, P. 2003, MNRAS, 345, 1271

Vermeulen et al. 1995, ApJ, 452, 5

Villata, M., et al. 2002a, A\&A, 390, 407

Villata, M., et al. 2002b, Mem. Soc. Astron. Italiana, 73, 1191

Villata, M., et al. 2004a, A\&A, 421, 103

Villata, M., et al. 2004b, A\&A, 424, 497

Villata, M., et al. 2009, A\&A, 501, 455 